\title{
Exploring solar-terrestrial interactions via multiple imaging observers
}

\section{G. Branduardi-Raymont, et al. [full author details at the end of the article]}

Received: 29 July 2020 / Accepted: 20 July 2021 / Published online: 16 August 2021

(C) The Author(s) 2021

\begin{abstract}
How does solar wind energy flow through the Earth's magnetosphere, how is it converted and distributed? is the question we want to address. We need to understand how geomagnetic storms and substorms start and grow, not just as a matter of scientific curiosity, but to address a clear and pressing practical problem: space weather, which can influence the performance and reliability of our technological systems, in space and on the ground, and can endanger human life and health. Much knowledge has already been acquired over the past decades, particularly by making use of multiple spacecraft measuring conditions in situ, but the infant stage of space weather forecasting demonstrates that we still have a vast amount of learning to do. A novel global approach is now being taken by a number of space imaging missions which are under development and the first tantalising results of their exploration will be available in the next decade. In this White Paper, submitted to ESA in response to the Voyage 2050 Call, we propose the next step in the quest for a complete understanding of how the Sun controls the Earth's plasma environment: a tomographic imaging approach comprising two spacecraft in highly inclined polar orbits, enabling global imaging of magnetopause and cusps in soft X-rays, of auroral regions in FUV, of plasmasphere and ring current in EUV and ENA (Energetic Neutral Atoms), alongside in situ measurements. Such a mission, encompassing the variety of physical processes determining the conditions of geospace, will be crucial on the way to achieving scientific closure on the question of solar-terrestrial interactions.
\end{abstract}

Keywords Solar wind $\cdot$ Magnetosphere $\cdot$ Ionosphere $\cdot$ Coupling $\cdot$ Global imaging $\cdot$ In Situ measurements

\section{Introduction}

The central question we propose to address is: How does solar wind energy flow through the Earth's magnetosphere, how is it converted and distributed? This is a fundamental science question expressing our need to understand how the Sun creates the heliosphere, and how the planets interact with the solar wind and its magnetic field. This is not just a matter of scientific curiosity - it also addresses a clear and pressing practical problem. As our world becomes ever more dependent on complex technology 
- both in space and on the ground - society becomes more exposed to the vagaries of space weather, the conditions on the Sun and in the solar wind, magnetosphere, ionosphere and thermosphere that can influence the performance and reliability of technological systems and endanger human life and health.

This fundamental question breaks down to several sub-questions: (1) How is energy transferred from the solar wind to the magnetosphere at the magnetopause? (2) What are the external and internal drivers of the different magnetospheric regimes? (3) How does energy circulate through the magnetotail? (4) How do behaviours in the North and South hemispheres relate to each other? (5) What are the sources and losses of ring current and radiation belt plasma in the inner magnetosphere? (6) How do feedbacks from the inner magnetosphere and ionosphere influence dayside and nightside processes?

Much knowledge has already been acquired through observations in space and on the ground over the past decades, but the infant stage of space weather forecasting demonstrates that we still have a vast amount of learning to do. We can tackle this issue in two ways: (1) By using multiple spacecraft (e.g. Cluster, THEMIS, Swarm, MMS) measuring conditions in situ in the magnetosphere in order to make sense of the fundamental small scale processes that enable transport and coupling, or (2) By taking a global imaging approach to observations of the conditions that prevail throughout geospace in order to quantify the global effects of external drivers.

Missions that had an element of global approach were IMAGE (Burch 2000) and TWINS (McComas et al. 2009), with the latter in particular performing multispacecraft ENA imaging of the ring current and plasmasphere, and also extending this to the magnetotail. A global approach is now being taken by a number of space missions under development, e.g. SMILE (Branduardi-Raymont et al. 2018) and LEXI (http://sites.bu.edu/lexi/), and the first tantalising results of their exploration will be available in the next decade. Here we propose the next step-up in the quest for developing a complete understanding of how the Sun gives rise to and controls the Earth's plasma environment: a tomographic imaging approach which can be achieved with an M-class mission consisting of two spacecraft enabling global imaging of magnetopause and cusps, auroral regions, plasmasphere and ring current, alongside in situ measurements. Such a mission will be crucial on the way to achieving scientific closure on the issue of solar-terrestrial interactions.

In this paper we present the science questions that need addressing (Section 2) and the observing methods that can be adopted (Section 3); Section 4 introduces the validation of magnetospheric models and in Section 5 we describe a space mission that could tackle the science questions raised in Section 2. In Section 6 we point out that such a mission does not involve technological challenges and we give our conclusions in Section 7.

\section{Science questions to be addressed}

The Earth's magnetic field extends into space (see Fig. 1), where its interaction with the supersonic solar wind plasma leads to the formation of the magnetosphere. The solar wind flow compresses the sunward side of the magnetosphere but drags the nightside out into a long magnetotail. A collisionless bow shock stands upstream from the 
magnetopause in the supersonic solar wind. The shocked solar wind plasma flows around the magnetosphere inside the magnetosheath.A relatively sharp transition from dense, shocked, solar wind plasmas to tenuous magnetospheric plasmas marks the magnetopause. High latitude cusps denote locations where field lines divide to close either in the opposite hemisphere or far down the magnetotail. Open field lines within the cusps provide an opportunity for solar wind plasma to penetrate deep into the magnetosphere, all the way to the ionosphere. The position and shape of the magnetopause change constantly as the Earth's magnetosphere responds to varying solar wind dynamic pressure and interplanetary magnetic field orientations. A basic component of magnetospheric activity is the Dungey cycle (Dungey 1961), illustrated in Fig. 2: intervals of dayside reconnection under southward interplanetary magnetic field (IMF) enable the magnetic flux content of the magnetotail lobes to increase and energy to be stored. Reconnection in the tail and magnetospheric convection bring plasma back to the dayside magnetosphere. The stored energy is intermittently and explosively released in geomagnetic substorms, and is associated with bright auroral displays in polar regions (e.g. Angelopoulos et al. 2008). Geomagnetic storms, usually driven by coronal mass ejections (CMEs) or corotating interaction regions (CIRs) (Borovsky and Denton 2006), and associated with prolonged periods of strong southward IMF, represent a severe space weather threat with the greatest capacity to disrupt everyday life throughout the world (e.g. affecting satellite subsystems and wellbeing in space, telecommunications, navigation, electrical infrastructures, and pipelines on the ground).

The energy input from the solar wind is continuously converted from the dynamic to the electromagnetic form and back, while plasma is moving through the magnetospheric

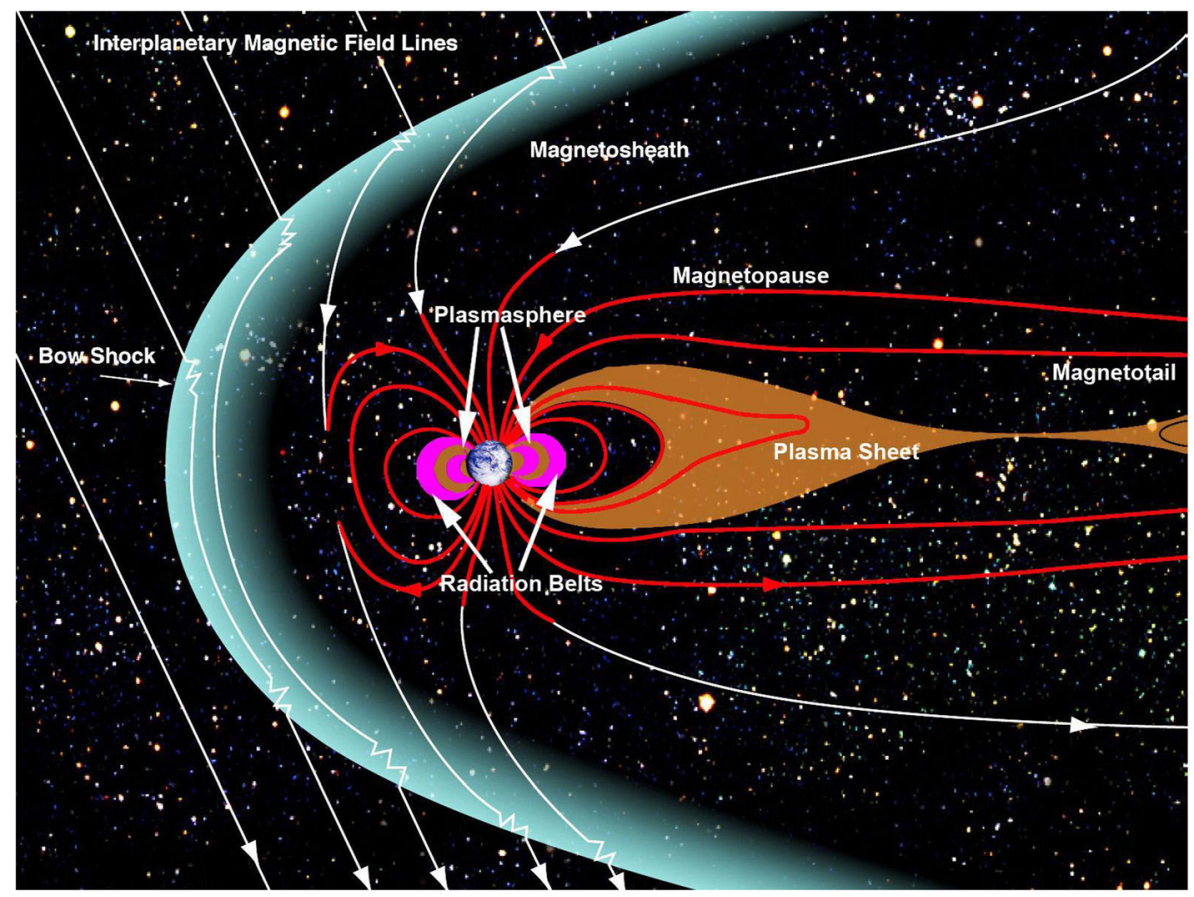

Fig. 1 The Earth's magnetosphere. (NASA/GSFC) 

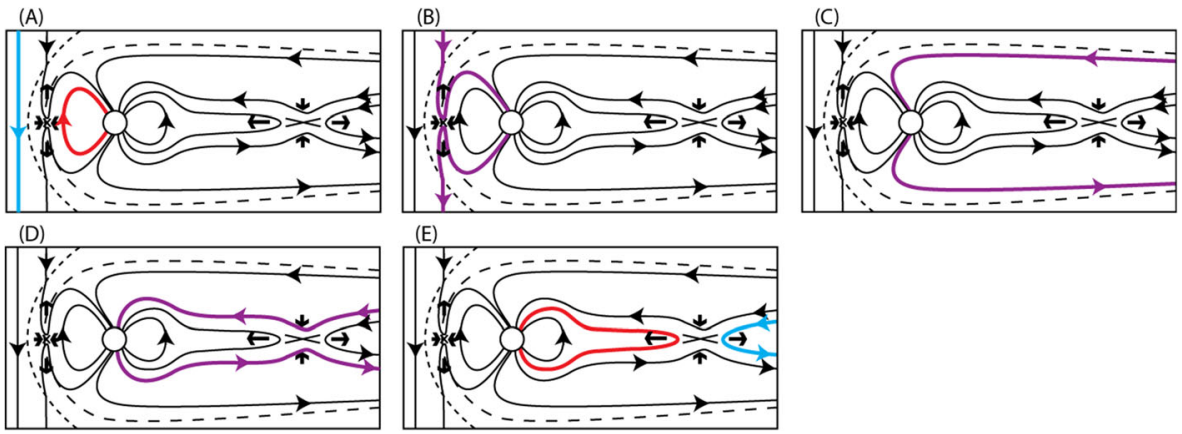

Fig. 2 Progression of the Dungey cycle. (A): Dayside reconnection opens magnetic flux (B) which convects over the poles and is stored in the magnetotail $(\mathbf{C})$ until an explosively release $(\mathbf{D})$ returns closed flux to Earth in conjunction with dramatic auroral displays (E) (from Eastwood et al. 2015)

and ionospheric regions (Fig. 2). The solar wind kinetic energy is partly converted to electromagnetic energy at the bow shock. Magnetic reconnection at the magnetopause plays a crucial role in the energy flux penetration from the solar wind into the magnetosphere (e.g. Cowley 1982), and this represents an opposite energy conversion process from the electromagnetic to the dynamic form. Due to the dayside magnetic reconnection, the magnetic energy is transmitted to the nightside magnetosphere, accumulated in the magnetotail and then suddenly released by nightside reconnection. The energetic particles from the magnetotail move towards the Earth, form the ring current and partly precipitate in the auroral regions. The precipitated particles and magnetospheric-ionospheric fieldaligned currents deliver the solar wind energy into the ionosphere. Ionospheric outflow constitutes an opposite mass and energy stream from the ionosphere into the magnetosphere, which influences nightside and dayside reconnection rates.

\subsection{Magnetospheric modes}

Depending on solar wind driving, the magnetosphere can change between different dynamic regimes, or magnetospheric modes, e.g. stationary magnetospheric convection, saw-tooth oscillations, isolated substorms and sequences of substorms and storms (e.g. DeJong et al. 2009; Pulkkinen et al. 2010, Sergeev et al. 1996; Walach et al. 2017, Hubert et al. 2017).

Transitions between the modes may result from changes in the upstream solar wind conditions and/or be consequences of the internal magnetospheric-ionospheric dynamics. In the first case, the IMF as well as solar wind plasma parameters regulate the energy input into the magnetosphere. The direction of the IMF plays an important role in the coupling between the solar wind and the magnetosphere. For example, during northward IMF conditions, coupling is complex, consisting of lobe reconnection as well as plasma transfer and energy/momentum input due to Kelvin-Helmholtz waves at the magnetospheric flanks (Taylor et al. 2008, Otto and Fairfield 2000). Quasi-radial IMF results in the foreshock formation in the dayside region, high-speed jets in the magnetosheath and magnetopause deformation (Plaschke et al. 2018). However, magnetic activity in the magnetosphere is stronger during southward IMF, when the IMF merges with the Earth's magnetic field at the dayside magnetopause. On the other hand, magnetospheric modes may be a consequence of internal magnetospheric - ionospheric 
dynamics. For example, Brambles et al. (2011) reported that the ionospheric $\mathrm{O}^{+}$ outflows can generate saw-tooth oscillations. While the different modes of magnetospheric dynamics are recognised, the questions of what external and internal conditions drive a particular mode and under what conditions mode transformations occur are still open. These are very significant issues in space weather research and have a strong bearing on future successful forecasts of the magnetospheric state and dynamics. Such issues are especially important as humans increasingly depend on technological infrastructures which can be adversely affected by space weather.

Global simulations, in situ and remote measurements have provided evidence for different reconnection modes at the magnetopause. Reconnection can be steady (Sonnerup et al. 1981) or bursty (Russell and Elphic 1978). The extension of this process across the dayside and flank magnetopause is also unclear. Understanding the temporal and spatial properties of magnetopause reconnection (e.g. variable solar wind driving, temporal behaviour of the reconnection process, length of reconnection line) is essential as they define how much energy is transferred from the solar wind into the magnetosphere. The energy return flow from the tail towards inner magnetosphere and ionosphere depends on the properties of substorms, which are another area of active research. Magnetospheric substorms may be triggered by solar wind variations and by either southward or northward IMF turnings, albeit with different efficiencies (e.g. Liou et al. 2003, Wild et al. 2009). On the other hand, some substorms occur without apparent IMF perturbations (Hsu and McPherron 2004).

Magnetospheric storms can be considered as additional, extreme, states of the Earth's magnetospheric dynamics, which are often the consequence of extreme heliospheric drivers, CMEs and CIRs (e.g. Denton et al. 2006). They are expected to occur on average at least once a month (McPherron1995). A storm is characterised by radiation belts build-up (although also depletion in some cases, Turner et al. 2013) and development of a strong ring current; however, it can also be accompanied by a number of other phenomena, e.g. by a series of substorms, by the motion of the auroral oval equatorward, strong and complex field-aligned currents and high fluxes of energetic particles. The magnetospheric dynamics during storm time is a subject of space weather research, and forecast of such dynamics is crucial for protecting satellites, ground infrastructure, and human health. It is now understood that the strongest storms are driven by CME/CME or CME/CIR interactions (Liu et al. 2015). However, internal magnetospheric processes are less clear, especially the role of the substorms in the development of the storm time ring current is still under active debate (e.g. Runge et al. 2018, Keller et al. 2005).

All the above illustrates that we have acquired qualitative knowledge about magnetospheric dynamics, e.g. about southward IMF triggering reconnection at the magnetopause, subsequent reconnection in the tail and how this affects the ring current during storms. However, we do not know how to quantify the energy circulation in the magnetosphere, we do not have precise knowledge of when and how the system changes as a result of given inputs, hence we are currently unable to predict strength and temporal variations of the storms and substorms. 


\subsection{Magnetopause and cusps}

The solar wind propagates through the bow shock and magnetosheath and interacts with the dayside magnetopause. During southward IMF intervals, the IMF reconnects with the magnetospheric magnetic field at the dayside magnetopause, and solar wind energy enters the magnetosphere. This results in reconfiguration of the magnetospheric - ionospheric (MI) currents, and the magnetopause in the subsolar region shifts Earthward (both empirical and numerical magnetohydrodynamics (MHD) models confirm this Earthward motion, e.g. Shue et al. 1998, Siscoe et al. 2002). The ground PC (Polar Cap) index also indicates when IMF discontinuities reach the subsolar magnetopause and the merging electric field at the magnetopause changes (e.g. Troshichev et al. 2006; Samsonov et al. 2017). Moreover, southward IMF and strong solar wind dynamic pressure applied to the dayside magnetopause cause global intensification of the aurora which can be observed for example at FUV wavelengths (e.g. Boudouridis et al. 2007). This almost immediate response to solar wind dynamic pressure variations is not connected with the energy accumulation in the magnetotail, instead energy and mass come directly from the dayside magnetopause. Energetic ions delivered through reconnection at the magnetopause increase the energetic ion population in the dayside and near-Earth magnetosphere (Luo et al. 2017).

Coupling of the solar wind with the magnetosphere also takes place through the magnetospheric cusps, where solar wind particles travelling along magnetic field lines can penetrate directly into the magnetosphere and ionosphere. The peculiar magnetic topology of the cusps means that they play a pivotal role in magnetospheric dynamics: they are the sole locations where solar wind has direct access to low altitudes (e.g. Cargill et al. 2005). During magnetopause reconnection, solar wind energy, mass and momentum are transferred through the cusps into the magnetosphere. The equatorward boundary of the cusp region is often identified as the boundary between closed dayside field lines and open ones. For southward IMF the cusp lies on the open field lines because of dayside magnetopause reconnection. The amount of open flux depends on the rate of reconnection both on the dayside magnetopause and in the nightside magnetotail plasma sheet, as well as on the extension of the $\mathrm{X}$-lines. Therefore, the cusps move to lower and higher latitudes as the open field line region expands and contracts, respectively. It is thus of key importance to continuously measure how the cusps respond to northward and southward turnings of the IMF, since this is intimately related to the strength of the solar wind magnetosphere coupling. Since the cusps are the endpoints of a large portion of the magnetospheric magnetic field lines, their structure, azimuthal extent, local time, and latitudinal location give information about a larger context than any other structure within the magnetosphere (see Sibeck et al. 2018 for a review). Since component and antiparallel reconnection models predict different cusp locations (Trattner et al. 2007), by studying the cusps we gather deeper insight into magnetopause reconnection.

Although multipoint in situ measurements of the cusps have provided evidence for the proposed reconnection mechanisms (e.g. stationary or temporal, component or antiparallel), global imaging can distinguish between them, quantify their significance, and also establish any asymmetry between North and South cusps. 


\subsection{Magnetotail reconnection and aurora formation}

Magnetotail reconnection begins when a significant magnetic energy has been stored in the magnetotail. The amount of the accumulated energy can be estimated by the size of the polar cap (Shukhtina et al. 2005). The area of open flux within the polar cap changes directly in response to the amount of open flux in the magnetotail lobes, which are defined by the dayside and magnetotail reconnection processes. Auroral activity and variations of the ground magnetospheric indices (e.g. SuperMAG indices, such as SME, SMU, SML; for acronyms see Newell and Gjerloev 2011) are the signatures of the subsequent energy release in the ionosphere and characterise substorm dynamics through variations of the MI currents. As field aligned currents provide coupling between the magnetosphere and ionosphere, the variability of the MI currents describes substorm dynamics.

The THEMIS mission (Angelopoulos 2008) was aimed at answering the key questions of magnetotail substorm timing and whether reconnection at the near-Earth $\mathrm{X}$-line is initiated first and drives the current disruption closer to the Earth, or the substorm starts with current disruption at $\sim 10-12 \mathrm{R}_{\mathrm{E}}$ and this launches the reconnection down the tail. However, results from THEMIS have shown that the magnetotail processes are more complex and the questions posed have not been answered in full.

Global simulations, in situ and remote measurements provide evidence for a wealth of magnetotail reconnection modes (e.g. McPherron et al. 2008; Walach et al. 2017; Dejong et al. 2009). For example, isolated substorms (e.g. Akasofu 2017) require a period of energy storage which can be initiated by southward IMF turnings. These isolated tail modes exhibit $\mathrm{a} \sim 1 \mathrm{~h}$ growth phase corresponding to an expansion of the auroral oval leading to a transient onset and auroral brightening. This is followed by a $\sim 30$ min expansion phase characterised by dynamic aurora moving poleward and stretching $\sim 3 \mathrm{~h}$ of Local Time across the nightside sky, and by strong electric fields in the inner nightside magnetosphere. The expansion phase is followed by $\mathrm{a} \sim 1 \mathrm{~h}$ recovery phase during which the aurora dims.

Figure 3 shows FUV imaging of the Northern polar cap by IMAGE for a six day interval which encompasses two storms (panel d) and multiple substorms. An estimate of the size of the polar cap can be derived from the radius of the auroral oval. These measurements have necessarily been non-continuous in the past due to the orbits of auroral imaging missions (data gaps in panel c), hindering progress in fully understanding solar wind - magnetosphere coupling. Short time-scale variations in polar cap size correspond to substorms, but large discontinuities exist over some data gaps indicating that the storm behaviour is only partially captured. This is an area where the continuous (over $40 \mathrm{~h}$ ) auroral monitoring by SMILE for the first time will make unbroken determination of the rates of magnetic reconnection and the factors that influence these. However, SMILE (Branduardi-Raymont et al. 2018) cannot do conjugate monitoring of the aurorae to capture hemispheric asymmetries, which is particularly necessary under northward IMF and under IMF with a strong dusk - dawn component.

When the IMF is directed northwards, lobe reconnection takes place at high latitudes, tailwards of the cusps' openings. Under sufficiently dense solar wind conditions, the footprint of the reconnection site is visible as an auroral 'spot' within the 
noon-sector of the polar cap (Milan et al. 2000, Frey et al. 2002, Carter et al. 2020). Unlike southward IMF reconnection, lobe reconnection is not constrained to occur equally in the northern and southern hemispheres and indeed the interrelation of lobe reconnection in the two hemispheres is unknown. The cadence of auroral imagers flown in the past has not been sufficient to properly analyse the dynamics of cusp spots. Consequently, the conjugate nature of these cusp and substorm features is completely unknown as simultaneous observations of both hemispheres are extremely rare, due to

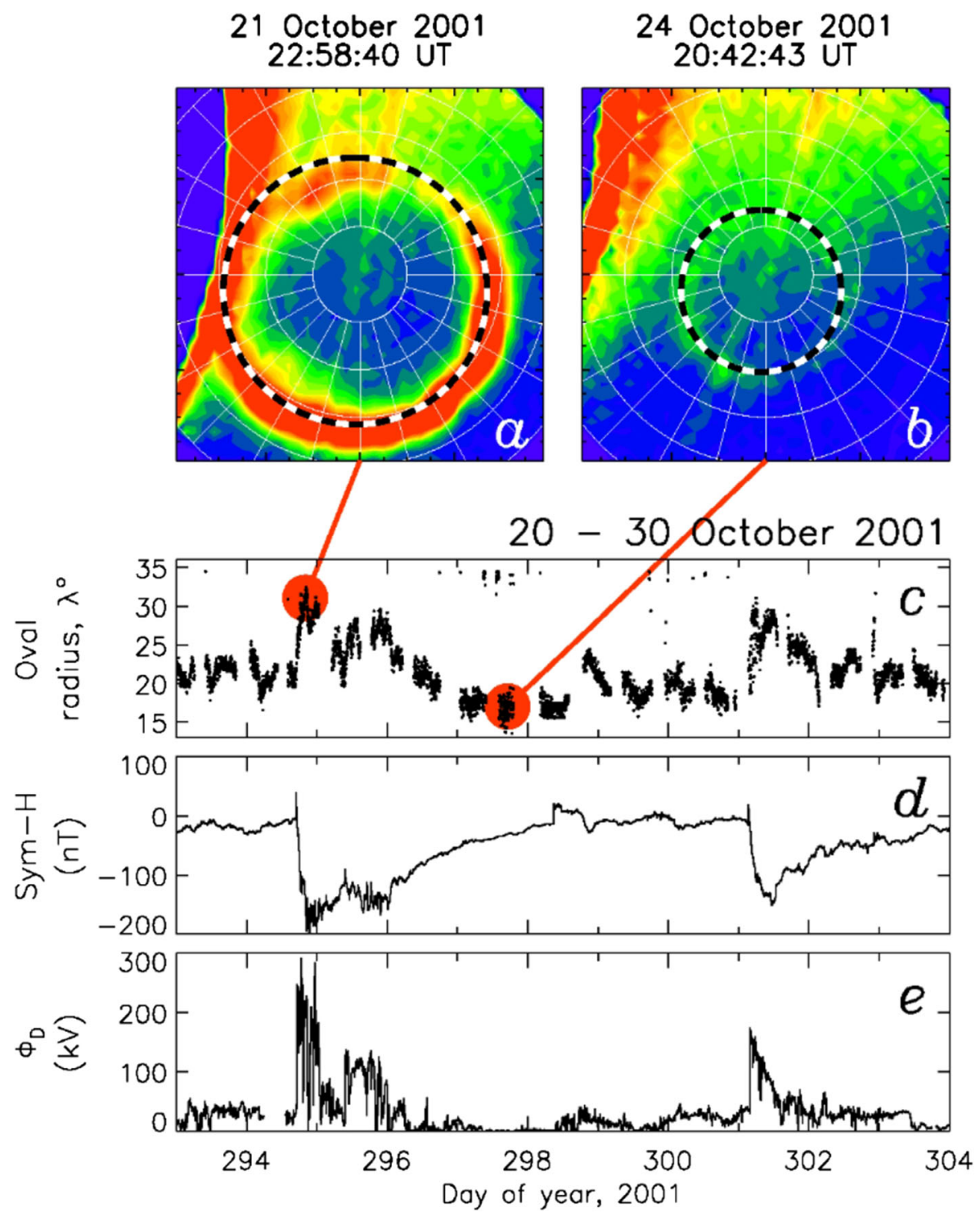

Fig. 3 (a and $\mathbf{b}$ ) The size of the polar cap is estimated from the radius of the auroral oval (images captured by the IMAGE satellite); (c, d, and e) Time-series of the radius of the auroral oval, the Sym-H index measure of ring current intensity, and a proxy for the dayside reconnection rate derived from upstream solar wind conditions (from Milan 2009) 
the lack of coordination between past missions. Other phenomena, such as transpolar arcs (TPAs), could be studied simultaneously in both hemispheres, and may react differently in each of them (Milan et al. 2005, Reidy et al. 2017, Østgaard et al. 2003, Carter et al. 2017).

Figure 4 illustrates simultaneous views of the North and South aurorae taken by the Polar and IMAGE spacecraft: some features are symmetrical, but many are not, challenging the current understanding of magnetic field mapping between hemispheres and our understanding of the fundamental processes leading to auroral emission. These may be the first clear observations of interhemispheric currents due to seasonal differences (Richmond and Roble 1987, Benkevich et al. 2000). Unfortunately, no global auroral imagers are in operation (the IMAGE mission ended in 2005), so true conjugate studies are not possible at the moment.

The very important but poorly understood issue of asymmetries in the magnetosphere - ionosphere field-aligned current system has not been adequately tackled so far. Significant open questions remain about the physics of substorm complete development, including its initialisation process. Such questions could be answered for the first time by continuous and conjugate FUV monitoring, with identical instrumentation, of both auroral ovals.

\subsection{Ring current sources and losses}

Energetic particles injected by the magnetotail reconnection contribute to the formation of the ring current. Energetic positively charged ions can undergo charge exchange with local neutrals turning into energetic neutral atoms (ENA), whose direction, energy, and species composition can be measured. By tracking the intensity of the created ENAs by charge exchange from the ring current as a function of time during isolated substorms, saw-tooth events, and storm-time substorms it is possible to quantify how individual events and prolonged intervals of nightside reconnection contribute to ring current plasma intensities, its sources and losses. ENA imaging (e.g. Mitchell et al. 2001, see Fig. 5) determines the depth to which ion injections penetrate, their azimuthal extent, the degree to which ions are energised, spectral slopes, and composition. ENA directly measures loss via charge exchange as a function of species $(\mathrm{H}, \mathrm{O})$, energy, location, and time. The second main contributor to the ring current formation is a large-scale electric field during stationary convective intervals in the nightside magnetosphere. The relative roles of the large-scale electric field and substorms in the buildup of the ring current are a subject of active debate (Keller et al. 2005).

Ring current ions with energies of $30-300 \mathrm{keV}$ at distances of $4-5 \mathrm{R}_{\mathrm{E}}$ on average from Earth originate in the Earth's plasma sheet. Each magnetotail reconnection mode supplies ions to the ring current. However, ring current intensities do not increase indefinitely. During storms, ring current intensities initially intensify, then decay, first rapidly over $\sim 8 \mathrm{~h}$ during the early recovery phase, and then more slowly over the next several days (Hamilton et al. 1988 - See Fig. 5). 


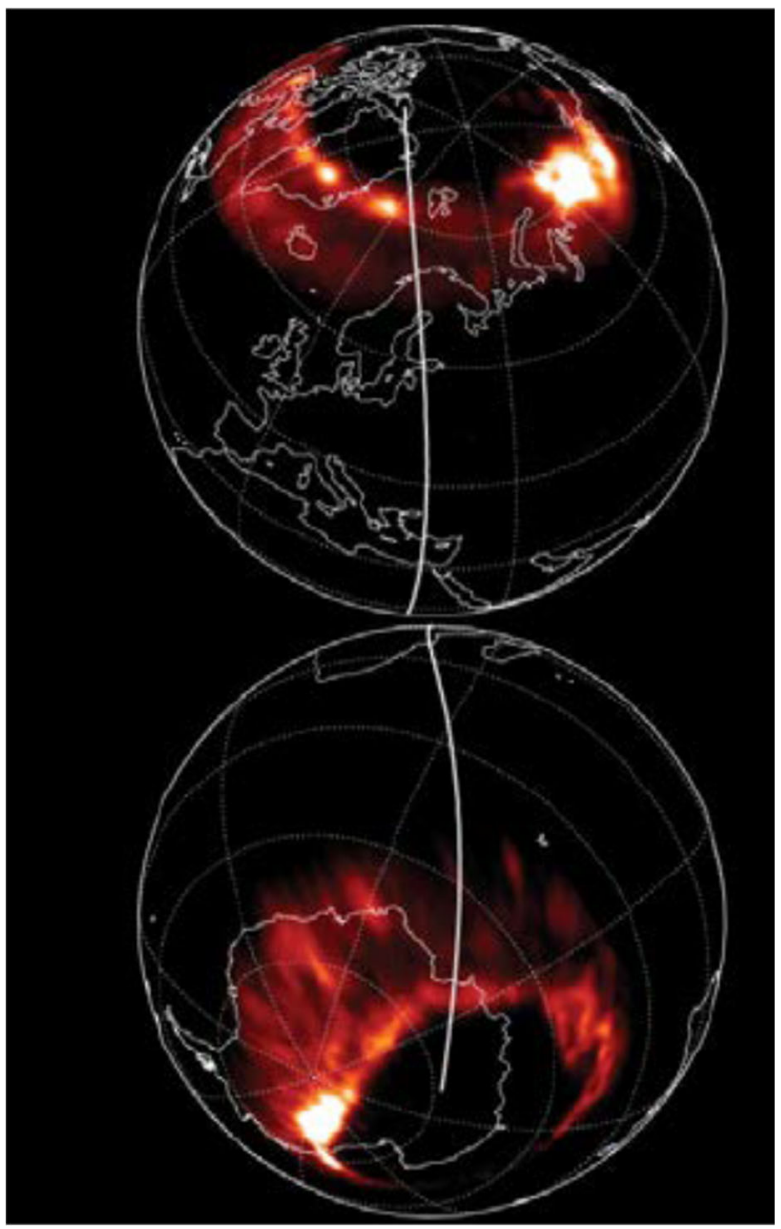

Fig. 4 Simultaneous views of the North and South aurorae obtained by Polar and IMAGE (from Laundal and Østgaard 2009)

Proposed mechanisms for ring current loss, in addition to charge exchange, include wave-particle induced precipitation and magnetopause outflow. Daglis et al. (1999) concluded that charge exchange with the exosphere is the main mechanism for ring current decay. As the convection electric field decays during the late recovery phase the slower charge exchange loss dominates ring current decay on completely closed drift paths (Takahashi et al. 1990, Liemohn et al. 2001). EMIC wave-particle interactions and precipitation may become important during the main phase of geomagnetic storms (e.g. Gonzalez et al. 1989). Significant dropouts of relativistic electrons can also take place as a consequence of magnetopause 'shadowing', following compression of the magnetopause, e.g. by an increase in solar wind dynamic pressure, and subsequent loss of trapped particles while drifting around the Earth (Herrera et al. 2016).

Despite the combination of modelling (e.g. Tsyganenko 2013) and observations (e.g. by TWINS: Goldstein and McComas 2013) we still have no good description of the 3-D shape of the ring current (especially during disturbed magnetospheric conditions), which is crucial in the $3-\mathrm{D}$ reconstruction of the magnetic field. Tomographic 

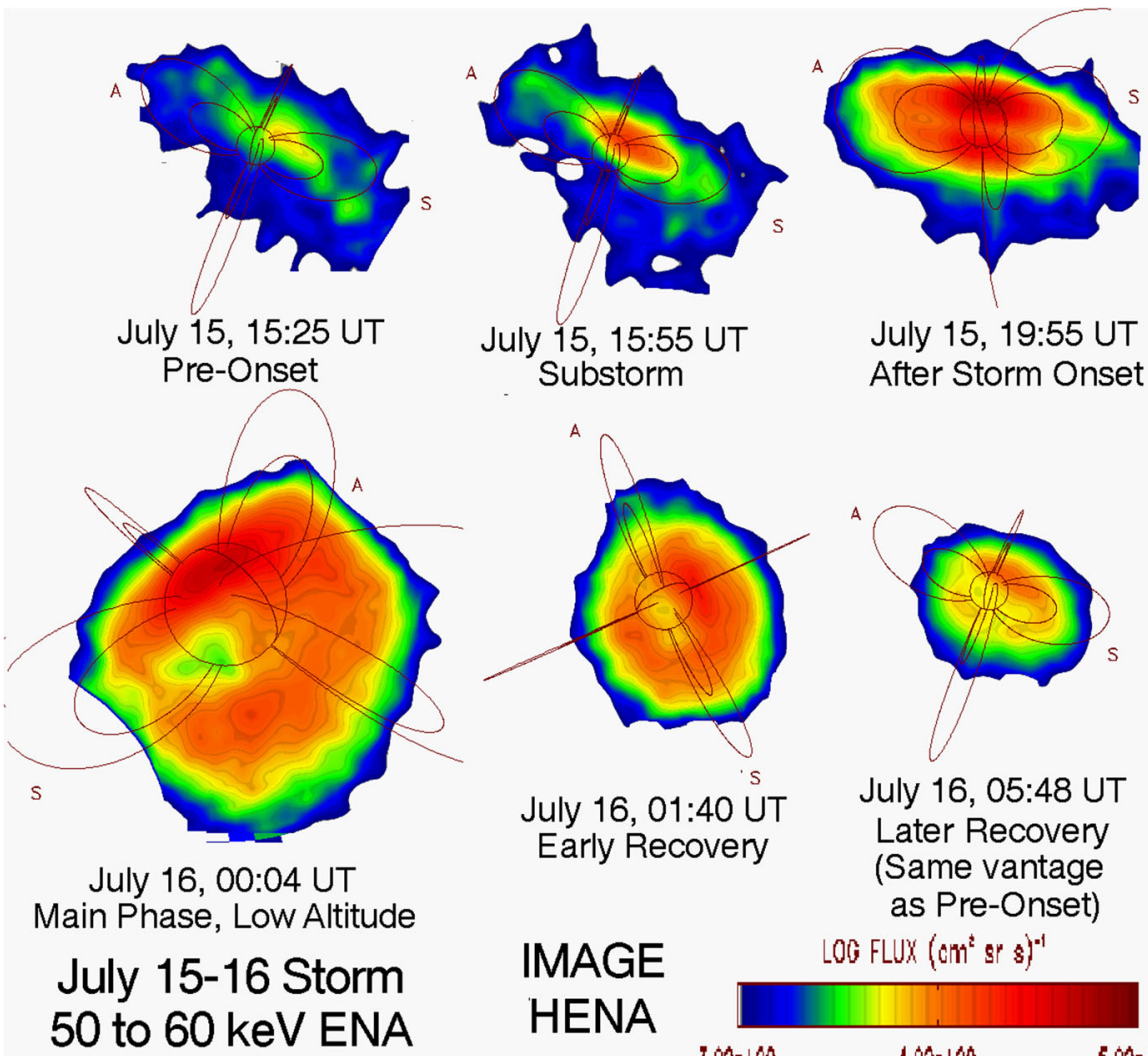

July 15, 19:55 UT After Storm Onset

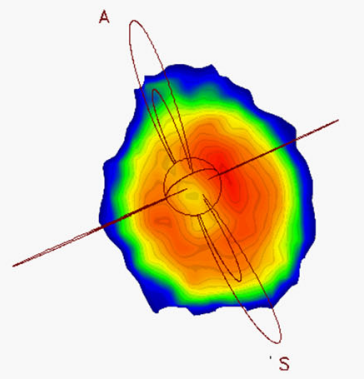

July 16, 01:40 UT Early Recovery

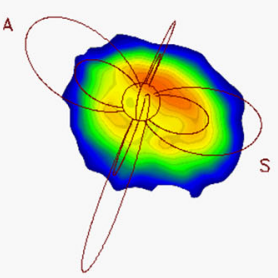

July $16,05: 48$ UT Later Recovery

(Same vantage as Pre-Onset)

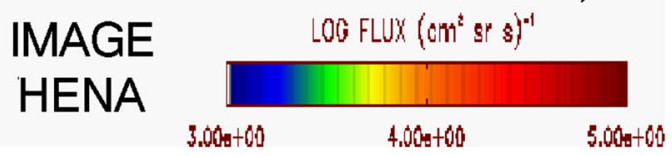

Fig. 5 ENA images of the fluctuation of Earth's ring current during July 15-16, 2000 geomagnetic storm (IMAGE HENA instrument, Mitchell et al. 2001)

measurements by ENA imagers mounted on at least two spacecraft on appropriately chosen orbits for the first time would provide the missing data.

Open questions are: (1) How efficiently the magnetotail response modes described above energise the ring current ions, (2) How transport and loss mechanisms affect the subsequent evolution of the ring current, (3) What is the relative importance of electric fields and substorms in building up the ring current, and of charge exchange, wave-particle interactions, and magnetopause shadowing in ring current losses. Determination of the 3-D structure of the ring current would help improve significantly our magnetic field models.

\subsection{Plasmasphere feedback}

The plasmasphere is a region of cold plasma of ionospheric origin which is trapped within the co-rotating portion of the inner magnetosphere. Its distribution has significant modifying effects on particle-particle and wave-particle interactions taking place in 
the inner magnetosphere. EUV emissions from the plasmasphere (see Fig. 6) help to interpret the feedback from the inner magnetosphere to the dayside magnetopause.

The size of the plasmasphere depends on the balance between co-rotation and convection, the latter depending on the strength of the solar wind interaction and reconnection in the magnetotail so that the plasmasphere expands during quiet conditions and contracts when the convection is strongly driven, e.g. during geomagnetic storms.

The inner magnetosphere is not simply the final stop in the circulation of energy in the coupled solar wind - magnetosphere system. Rather, the inner magnetosphere hosts dynamic processes that have important feedback effects on the solar wind-magnetosphere interaction.

Plasmaspheric plumes frequently become entrained in magnetopause reconnection (McFadden et al. 2008) particularly during geomagnetic storms when plumes persist for days (Borovsky and Denton 2008). Since reconnection rates are inversely proportional to plasma densities (Cassak and Shay 2007), the arrival of a high density plasmaspheric plume at the magnetopause quenches reconnection.

How the plasmasphere is refilled and how the plasma distributes itself along the field lines are still open questions, as well as the time-dependent processes that lead to plasmaspheric loss and to the transport of cold plasma within the magnetosphere.

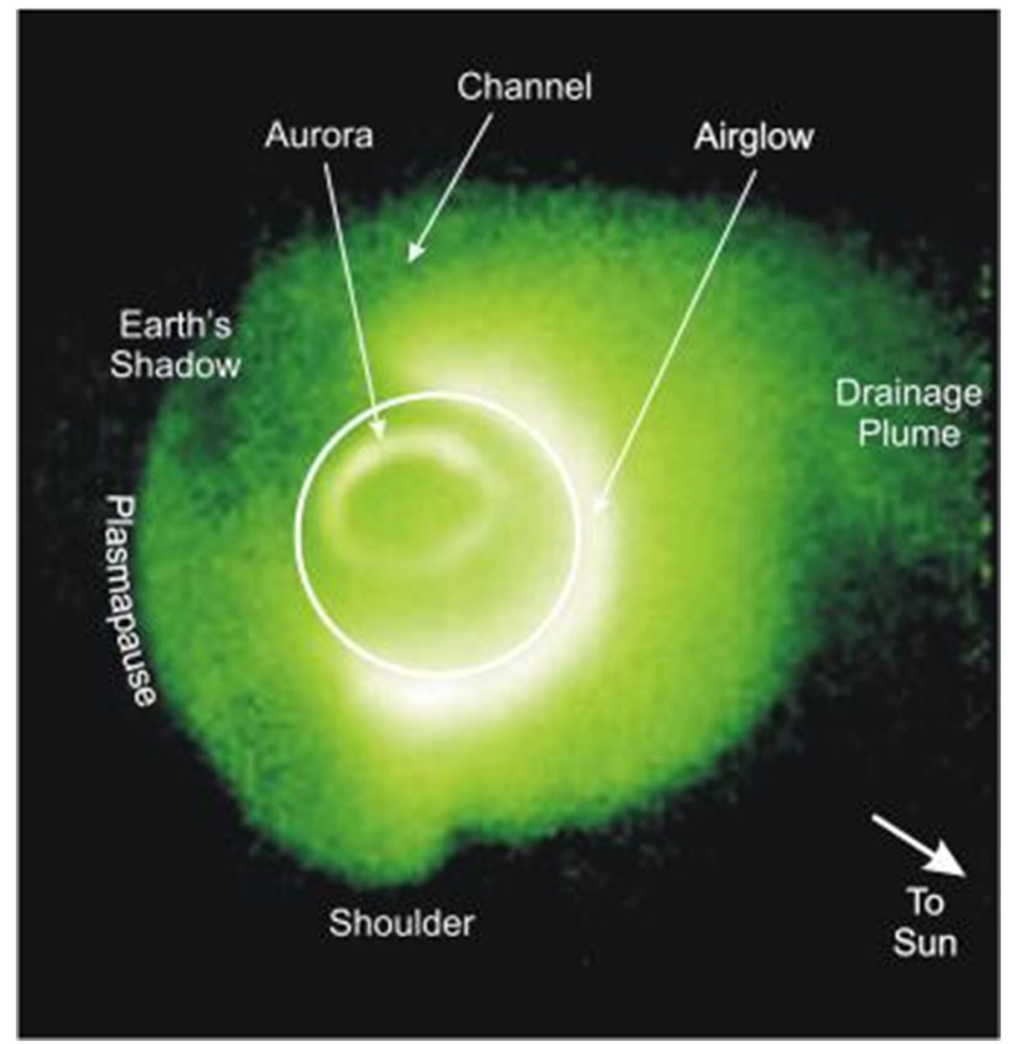

Fig. 6 Earth's plasmasphere and plume as measured by IMAGE's EUV instrument (from Sandel et al. 2003). The white circle represents the size of the Earth 


\section{Observing methods to answer open science questions}

\subsection{Magnetopause and cusps imaging}

Solar wind charge exchange is now recognised as the atomic process generating soft X-rays (0.2-2.0 keV, a range including a large number of $\mathrm{K}$ - and L-shell atomic transitions) in a variety of astrophysical scenarios, and in the Earth's magnetosheath and magnetospheric cusps too. This has enabled a novel approach to mapping the magnetosphere in a global way: wide field of view (FOV) X-ray images allow us to study kinetic physics in geospace on the global scale. So far applications of this technique (e.g. SMILE Soft X-ray Imager) have been limited to single imagers: these will return 2-D images from which the 3-D structure of the magnetopause needs to be reconstructed, with line of sight integration introducing uncertainties. These could be alleviated substantially by tomographic measurements performed by having imagers on at least two spacecraft on appropriately chosen orbits.

Soft X-ray images with high sensitivity and good spatial and temporal resolution are required to reach closure on some of the science questions stated above, e.g. Earthward magnetopause shifting for southward IMF; formation, dynamics, and properties of Flux Transfer Events, or FTEs; magnetopause motion after solar wind directional discontinuities; high-speed jets downstream of the quasi-parallel bow shock; magnetopause indentations, and possibly Kelvin-Helmholtz vortices.

\subsection{Auroral imaging}

Auroral emissions are produced by the precipitation of energetic electrons and protons. High energy precipitating electrons excite atmospheric constituents by impact, with emissions in the FUV band from Lyman-Birge-Hopfield transitions of $\mathrm{N}_{2}(140-180 \mathrm{~nm})$ and $\mathrm{OI}$ at $135.6 \mathrm{~nm}$. Electrons with energies in excess of $30 \mathrm{keV}$ produce X-rays by bremsstrahlung. Proton aurorae are seen at the footprints of the magnetospheric cusps, where they are injected from the magnetosheath by lobe reconnection (Frey et al. 2002) although their relationship to similar electron aurorae (Milan et al. 2000) is still unknown. Proton precipitation produces Ly $\alpha$ emission as protons charge exchange with atmospheric constituents to create excited $\mathrm{H}$ atoms. In order to distinguish the emission of (down-travelling) precipitating protons from the geocoronal Ly $\alpha$ the redshifted wing of the Ly $\alpha$ line is imaged. This requires a system that efficiently rejects both the geocoronal Ly $\alpha$ emitted at $121.6 \mathrm{~nm}$ and the NI multiplet at $120 \mathrm{~nm}$. IMAGE was able to image the proton aurora.

Observations of the dynamic aurora caused by energetic electron precipitation on the nightside (discrete auroral arcs) can be used for the study of the magnetotail processes and observations of the auroral oval in both hemispheres for the investigation of the global solar wind - magnetosphere coupling and its inter-hemispheric symmetries. Breakthrough science can arise from operating two three-axis stabilised spacecraft, allowing continuous and conjugate imaging of both aurorae, with high cadence imaging and the capability to suppress dayglow contamination. 


\subsection{Ring current imaging}

ENAs are produced when singly positively charged energetic ions undergo charge exchange collisions with cold neutral atoms or molecules. The ions become neutral and travel on unaffected by electromagnetic fields. In addition to carrying spectral and directional information of the energetic ions, the ENAs also provide direct measurement of their species composition. In the Earth's magnetosphere the ring current charge-exchanges with the geocorona at high altitudes, emitting ENAs that allow the ring current and plasma sheet ion populations to be imaged (Roelof et al. 2004).

ENA imaging maps the neutral hydrogen and oxygen ions generated when ring current and plasma sheet ions encounter exospheric neutrals, providing the information needed to track plasma sheet thinning and recovery, ring current growth and decay via substorm particle injections, precipitation, charge exchange, and magnetopause outflow. ENA imaging further identifies ring current effects on the magnetopause and nightside reconnection modes. Simultaneous monitoring of magnetopause and ring current allows to investigate directly and quantify magnetopause shadowing, i.e. the loss of energetic particles through the magnetopause when this is closer to the Earth, within the global loss of magnetospheric plasma.

\subsection{Plasmasphere imaging}

Global images of the plasmasphere can be obtained by observing EUV sunlight at $30.4 \mathrm{~nm}$ resonantly scattered from singly-ionised Helium, a minor magnetospheric constituent which allows extrapolation of overall magnetospheric density (Sandel et al. 2000). $\mathrm{He}^{+} 30.4 \mathrm{~nm}$ is the brightest ion emission from the plasmasphere, it is spectrally isolated, and the background at this wavelength is negligible. Measurements can be easily interpreted because the plasmaspheric $\mathrm{He}^{+}$emission is optically thin, so its brightness is directly proportional to the $\mathrm{He}^{+}$column abundance.

EUV imaging can track the plasmapause to quantify the convection that occurs in response to dayside and nightside magnetotail reconnection modes, to identify locations where wave-particle interactions may drive ring current ion precipitation, and to determine when and where plasmaspheric plumes may affect dayside reconnection. EUV observations of plasmapause motion distinguish between and quantify steady and impulsive electric fields applied to the inner magnetosphere.

\subsection{In situ measurements}

The imaging observations outlined above require to be set into context by the availability of simultaneous in situ measurements of plasma and fields. These could be gathered by monitoring spacecraft at the L1 point and propagated to near Earth orbit, although continuity and strict simultaneity cannot be assured. There are a few clear advantages of measuring immediately upstream as opposed to relying on L1. In situ monitoring of plasma conditions from locations outside the bow shock and in the Earth's vicinity, where remote imaging is performed, is a much more appropriate solution: especially if multiple spacecraft are involved, it quantifies the solar wind input and determines orientation and structure of solar wind discontinuities, arrival times at the magnetopause, and impact on the magnetosphere with much better 
accuracy than that available from propagating observations from a monitor at L1. If the spacecraft is in the foreshock then foreshock bubbles may be observed in situ and add context to the images (i.e. they could explain possible dawn-dusk asymmetries), which would otherwise be missed at L1. Also, interplanetary coronal mass ejections (ICMEs) are very dynamic and data at L1 do not take into account the expected evolution of the structures which may be important for triggering substorms.

Simultaneous in situ measurements in the northern and southern hemispheres (and on dawn and dusk flanks) can provide information about inclination of solar wind discontinuities and also about homogeneity of the solar wind stream (answering questions such as: Do we observe the same solar wind parameters on a separation of $40-60 \mathrm{R}_{\mathrm{E}}$ ?). Very useful measurements could be made in the magnetotail (since probably there will be two 'tail seasons' per year). These would include magnetic field strength in the lobes (closely related to the expanding/contracting polar cap and solar wind pressure effects), and magnetic field and particle measurements in the plasma sheet at down-tail distances, where the near-Earth reconnection line is expected to be located.

\subsection{Possible complementary observing methods}

The key magnetospheric processes resulting in energy transfer and partition, and the transient structures which they create, involve accelerated and energised plasma, hence coverage (by imaging and in situ measurements) at high energies (e.g. tens of $\mathrm{keV}$ and above) is the way to identify and characterise these processes. Looking relatively far into the future, global measurement requirements expected to cover higher energy ranges could include high energy X-ray imagers and also tracers for the energetic particles themselves as part of the in situ package, in order to understand coupling between regions.

\subsection{Ground measurements}

Ground-based all-sky imagers and magnetometers distributed throughout Canada, Alaska, and Northern Europe, and in Antarctica, are an essential complement to the space-borne instruments by providing the high time and spatial resolution observations needed to track the development of critical nightside auroral microstructures, including those that herald substorm onset. Global networks of ionospheric radars (e.g. SuperDARN, Super Dual Auroral Radar Network, Chisham et al. 2007) provide measurements of convection, which in turn quantifies reconnection rates (as well as the expanding/contracting polar cap). Combined space- and ground-based images (such as those from all-sky auroral cameras) place mesoscale auroral structures in their global context in a manner impossible during the IMAGE and THEMIS eras, and throughout almost the entire POLAR era.

\section{Validation of magnetospheric models}

An essential part of researching solar-terrestrial relationships is the development and validation of empirical and numerical magnetospheric models. Global magnetospheric 
conditions and dynamics can be simulated by means of numerical and empirical (and semi-empirical) models (Gordeev et al. 2015). A large number of models have already been developed for different magnetospheric regions (bow shock and magnetopause, magnetotail, inner magnetosphere, ionosphere and upper atmosphere, auroral regions). Some models are local and developed to simulate a particular region or for predicting some specific parameters, and some models are global and able to reproduce the global magnetospheric dynamics self-consistently, i.e. taking into account interrelations between different regions. The model development is important, because we can both better understand the physics of the processes and make predictions of extreme magnetospheric-ionospheric conditions which are important for space weather forecast.

International and national meteorological and space agencies have used some of these models already. In particular, for aurora forecast NOAA uses the OVATIONPrime model, which predicts global 2-D images of the auroral activity depending on solar wind conditions, while the University of Michigan's Geospace model can predict $\mathrm{Kp}$ (the geomagnetic activity index) and Dst (the disturbance storm time index) as well as regional magnetic variations, and the Relativistic Electron Forecast Model (REFM) is used for high-energy electron fluence at geosynchronous orbit (see www.swpc.noaa. gov). The Geospace model, a part of the Space Weather Modeling Framework (SWMF) developed at the University of Michigan, simulates the full time-dependent 3D geospace environment (Earth's magnetosphere, ring current, and ionosphere) and predicts global space weather parameters such as induced magnetic perturbations in space and on the Earth's surface. The current version of the Geospace model includes the global magnetospheric MHD model BATS-R-US and a kinetic model of the inner magnetosphere (Liemohn et al. 2018). The European space community has also developed several models e.g. the global kinetic model VLASIATOR by the Finnish Meteorological Institute (https://www.helsinki.fi/en/researchgroups/vlasiator/aboutvlasiator) and the TRANSPLANET ionospheric model (http://ransplanet.irap.omp. $\mathrm{eu} /$ ), and other space agencies have done the same, mostly for high-energetic particles and ionospheric disturbances, based predominantly on empirical models.

We anticipate that global magnetospheric models will become even more significant in the future because the magnetosphere is a region where processes in different parts are closely related to each other. One of the main approaches will be using combinations of fluid and kinetic models. These models can simulate both the propagation of the solar wind from the solar corona to the magnetosphere and its subsequent interaction with the magnetosphere and ionosphere, ultimately predicting geomagnetic disturbances and ionospheric conditions crucially important for human civilization and infrastructures. Another possible approach in the modelling is using artificial intelligence systems.

Irrespective of which one is used, any model has to be fully validated. However, this is not achievable at the present time. Most past and present space missions provide in situ measurements which cannot reflect the global magnetospheric configuration because the observations can be completely different when changing locations even slightly. Recent multi-spacecraft missions (Cluster, THEMIS, MMS) do not solve this problem completely because the observations are still sparse and local. Global context can be obtained by statistics, but this requires years of measurements with varying orbits (e.g. Cluster, THEMIS), and even then the coverage is limited for different solar wind conditions. The alternative of using many spacecraft is extremely expensive and 
impractical budget-wise for one agency. Imaging can produce global coverage almost immediately, for the immediate upstream conditions and at an affordable cost.

We only achieve some global observations near the Earth, e.g. using a large set of ground magnetometers and all-sky cameras; global magnetospheric imaging has been only sporadic so far. Using direct global measurements we can simultaneously validate both empirical models and combined MHD-kinetic models in several principal magnetospheric regions. Moreover, global imaging can underpin the development of a new generation of models which satisfy all observational constraints from the start.

Missions which use global imaging techniques have already been in operation but are no longer so (Polar, IMAGE), or are in preparation (SMILE, LEXI). However, to progress we need higher accuracy, better spatial resolution, and simultaneous coverage of different magnetospheric regions by using different techniques in order to reconstruct the global magnetospheric dynamics and validate future magnetospheric models. We also need stereo vision, i.e. simultaneous observations from at least two different locations, in order to reconstruct 3-D distributions of magnetospheric parameters from 2-D global images. All magnetospheric boundaries and structures are three-dimensional and they are not necessarily approximated by simple mathematical functions as often assumed. Our current descriptions of the magnetopause and ring current shapes are examples of such simplified interpolations. Using stereo vision, we can reconstruct the real shape of these boundaries and current layers without any a priori assumptions.

\section{How can a space mission address the open science questions?}

Remote global magnetospheric images together with in situ solar wind measurements near the bow shock and available ground magnetospheric indices (which reflect the intensity of main magnetospheric and ionospheric current systems as observed by a large set of ground magnetometers) will provide complete information about the magnetospheric state, its variations, and evolution. Using global magnetospheric imaging, we can collect simultaneously essential information about the positions and physical conditions of the main magnetospheric boundaries, i.e. the magnetopause, bow shock, magnetospheric cusps, the auroral oval, the ring current, and plasmapause. Using this full array of measurements, we obtain a complete description of the magnetospheric state and can distinguish different magnetospheric modes. We also can specify how the global magnetospheric physical state and positions of magnetospheric boundaries change when the magnetosphere switches from one mode to another.

The science questions raised in Sec. 2 have been explored to some extent by a number of multipoint missions such as Cluster, THEMIS, Van Allen Probes, and MMS, which target reconnection at the magnetopause, the substorm cycle, radiation belts transport and loss processes, the microphysics of reconnection and particle acceleration, and provide tantalising evidence for competing solar wind - magnetosphere interaction modes. However, they do not provide the global view necessary to 
distinguish between proposed interaction modes, determine their occurrence patterns and hence quantify their global significances.

In the near future the physics of solar-terrestrial interactions is going to be probed with dedicated space missions under development and planned (e.g. CuPID, launch 2021; LEXI, 2022; SMILE, 2024; see Sibeck et al. 2018). These apply the novel technique of soft X-ray imaging of the Earth's magnetosphere, usually coupled with well established FUV imaging of the auroral oval and in situ measurements. These missions will tackle some of the open questions, but will be unable to explore issues linking the solar wind magnetosphere coupling with the ring current and plasmasphere, and especially those issues where the combined global view from multiple vantage points is required (e.g. energy transformation between different magnetospheric and ionospheric regions, taking into account asymmetries between northern and southern hemispheres). Moreover, we can expect that the new knowledge provided by forthcoming missions will lead to new lines of investigation, requiring a step up in observing facilities in the longer term.

A comprehensive approach to studying solar-terrestrial interactions on the global scale could be achieved by a space mission capable of providing stereo vision for tomography studies of the Earth's magnetosphere: this would include X-ray imaging of the dayside magnetosheath and the magnetospheric cusps, coupled with simultaneous FUV monitoring of both North and South aurorae, imaging of the plasmasphere in the EUV and in ENA for the ring current. Using two spacecraft, 3-axis stabilised, in appropriately selected orbits, and two identical payloads, would allow significantly better reconstruction of the 3-D shape of the magnetopause, ring current, and plasmasphere. An integral part of each payload would also be an in situ instrument package dedicated to providing self-sufficient monitoring of solar wind and magnetosheath plasma conditions.

\subsection{Orbit(s)}

In order to achieve a good vantage point while maintaining appropriate spatial resolution a large geocentric distance (e.g. $30 \mathrm{R}_{\mathrm{E}}$ ) is suggested (a lower distance would be advantageous for auroral imaging at high resolution, but would hinder the tomography capability).

Two circular, highly inclined, polar orbits, with a 9.65 day period and phased $90^{\circ}$ away from each other, would allow observations of magnetospheric plasma structures and the solar wind input from both polar and equatorial vantage points and achieve the goals of tomography and conjugate auroral monitoring discussed above. The $90^{\circ}$ phasing, unlike $180^{\circ}$, allows simultaneous continuous monitoring of both aurorae, although only twice an orbit at nadir. Figure 7 shows simulated side and polar views of the magnetosphere from a $30 \mathrm{R}_{\mathrm{E}}$ orbit using the CCMC (Community Coordinated Modeling Center) BATS-R-US model.

A study by the NASA GSFC (Goddard Space Flight Center) members of this proposal team has shown that a $30 \mathrm{R}_{\mathrm{E}}$ circular polar orbit can be reached with lunar assist; two spacecraft, properly phased, can observe simultaneously both aurorae for long intervals over the 9.5 day orbital period, with the added bonus that circular orbits do not need adjusting. For some orbits, simultaneous 
observations on the dayside and nightside may also be possible, and, in some cases, crossing the bow shock from the solar wind into the magnetosheath, allowing us to measure different regimes.

\subsection{Payload}

\subsubsection{X-ray imager}

An enhanced version of the SMILE Soft X-ray Imager (Branduardi-Raymont et al. 2018, and Fig. 8) can be envisaged.

From a geocentric distance of $30 \mathrm{R}_{\mathrm{E}}$ it is possible to capture the entire dayside magnetopause within a $30^{\circ}$ x $30^{\circ}$ FOV without sacrificing spatial resolution: Fig. 9 shows simulated soft X-ray images corresponding to observations made from two spacecraft with $90^{\circ}$ phase shift and slightly different aim points in order to observe a larger area.

This is based on low mass lobster-eye optic technology, combined with large frame CCD or microchannel plate detecting devices. The former detectors provide energy resolution $(\sim 50 \mathrm{eV}$ at $0.5 \mathrm{keV}$ with current technology) but are susceptible to radiation damage, while the latter offer no spectral resolution, are less radiation sensitive and require high voltages.

Given the current detector developments for X-ray astrophysics missions, it is likely that the detector of choice for a future mission will be an active-pixel sensor using CMOS technology, offering all the performance benefits of CCDs with increased radiation hardness.

\subsubsection{Auroral FUV imager}

The auroral imager requirements can be derived from experience with previous missions (e.g. Polar, IMAGE) and the development of the SMILE UV Imager. The new science emerges from the fact of having two spacecraft, permitting continuous and conjugate imaging, and spacecraft that are 3-axis stabilised, allowing continuous pointing and high cadence imaging with long exposure times.
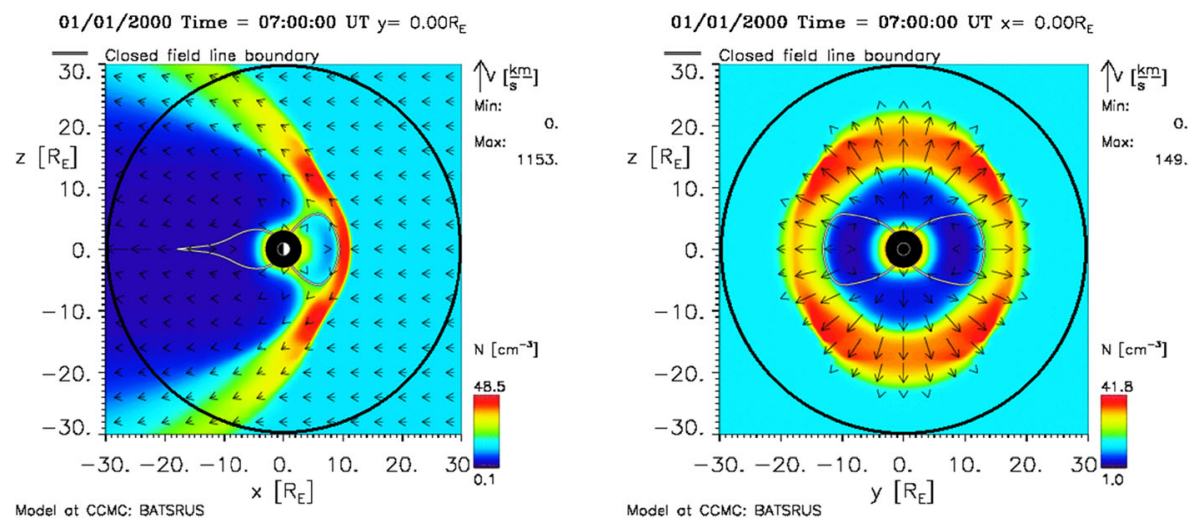

Fig. 7 MHD simulations of the plasma conditions and viewing from a 30RE polar orbit 


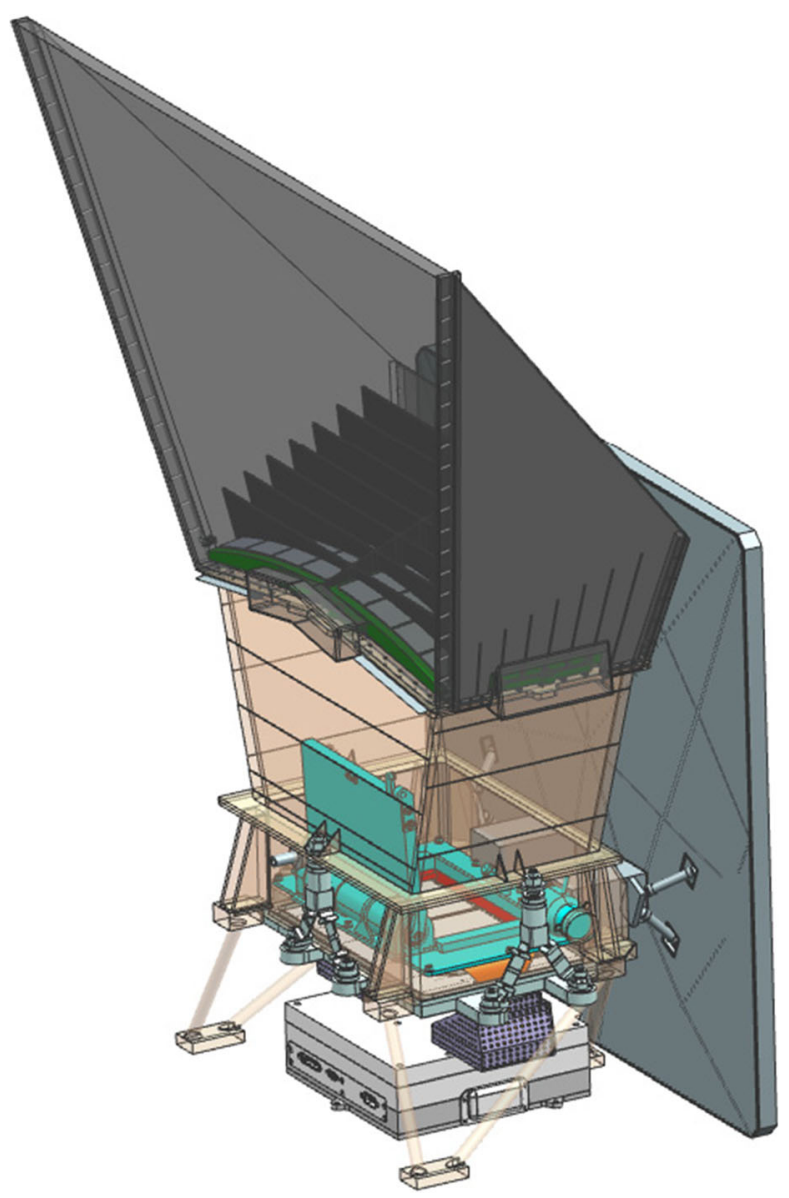

Fig. 8 CAD model of the SMILE Soft X-ray Imager and (bottom) Front End Electronics (BranduardiRaymont et al. 2018)

The ability to suppress non-auroral emissions is vital for the science objectives, since the dayside aurora will be sunlit in at least one of the hemispheres most of the time (see Fig. 10 for an example from IMAGE WIC). Deposition of thin-film reflective coatings on mirrors with several reflecting surfaces, as well as on the detecting devices, enable isolation of the desired FUV passband for electron aurora (e.g. 160-180nm), and provide many orders of magnitude visible light suppression. Alternative instrument designs can be considered for proton aurora imaging (at Ly $\alpha$, e.g. Mende et al. 2000). A $5^{\circ} \times 5^{\circ} \mathrm{FOV}$ is appropriate at a geocentric distance of $30 \mathrm{R}_{\mathrm{E}}$.

The spatial resolution needs to be of the order of $100 \mathrm{~km}$, similar to previous imaging missions, with a cadence of $2 \mathrm{~min}$.

\subsubsection{ENA imager}

ENAs are generated in the terrestrial magnetosphere through charge exchange processes between magnetically trapped energetic ions and cold neutral gas. An ENA camera 

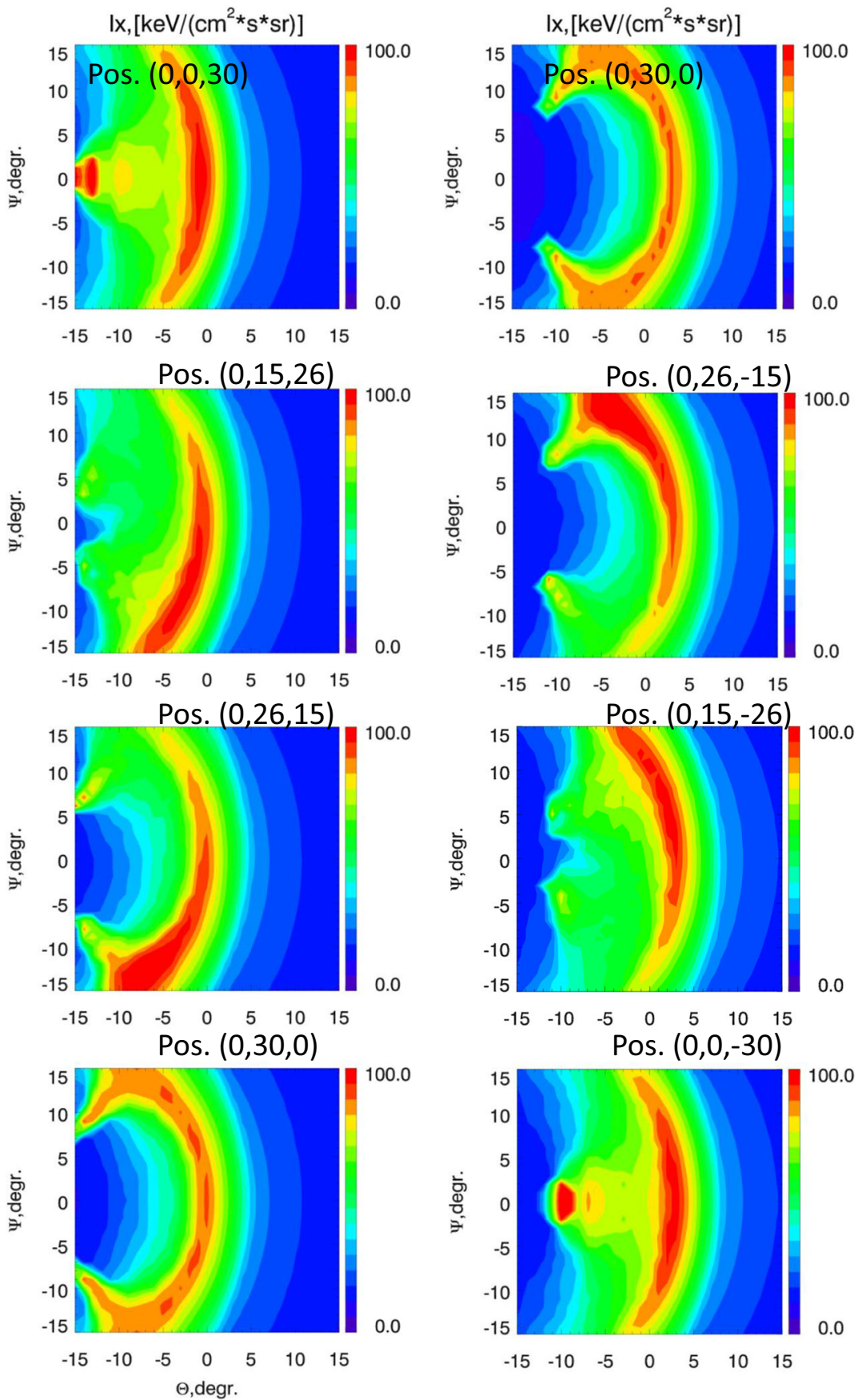

Fig. 9 Simulated soft X-ray images corresponding to observations made from two spacecraft at different points in the orbit (indicated over each panel) and with different aim points (all in GSM coordinates) 

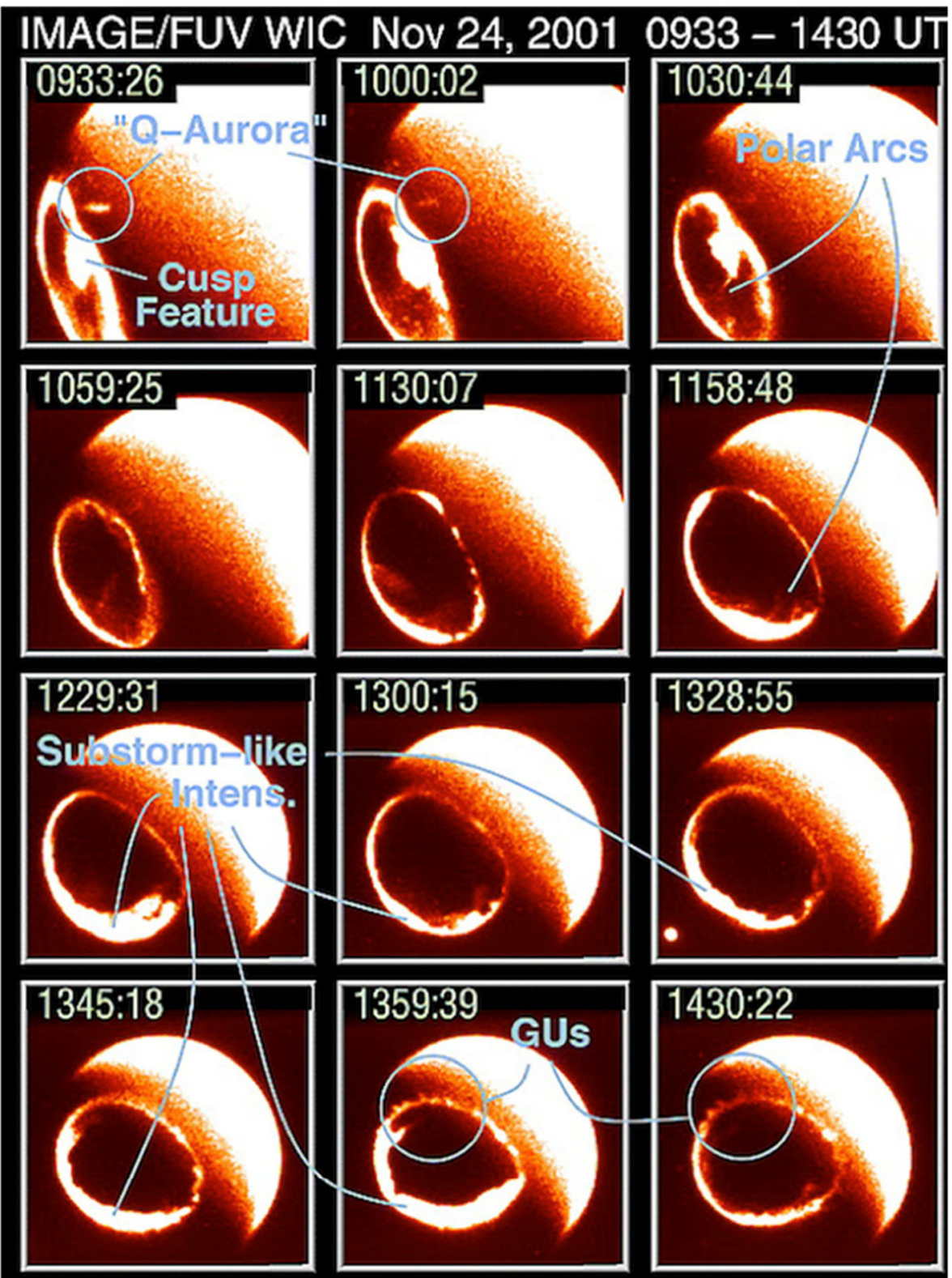

Fig. 10 IMAGE WIC FUV imaging: Northern aurora during a substorm (NASA/GSFC)

can record the arrival directions, energies, and mass species of magnetospheric ENAs as well as indirectly provide global images of the spatial, energy, and mass species distributions of their parent ion populations.

Figure 11 shows a schematic of the IMAGE HENA instrument, with a FOV of $120^{\circ}$ $\mathrm{x} 90^{\circ}$, covering the energy range $20-500 \mathrm{keV}$ per nucleon, with energy resolution $\Delta \mathrm{E} /$ $\mathrm{E}<0.25$ and velocity resolution of $50 \mathrm{kms}^{-1}$. After an electrostatic deflection assembly 
at several $\mathrm{keV}$ positive potential has prevented charged particles from entering the camera, the remaining ENAs pass through a Time of Flight device which allows to compute their incoming direction and their velocity. The energy deposited in a solid state detector (SSD in Fig. 11) enables mass discrimination (separation of hydrogen and oxygen atoms).

A spatial resolution of a couple of $\mathrm{R}_{\mathrm{E}}$ and cadence of $30 \mathrm{~min}$ are adequate. Similar requirements apply to the study of the plasma sheet.

\subsubsection{Plasmasphere EUV imager}

The imaging requirements can be derived from heritage of the EUV instrument flown on IMAGE, which was spinning and carried three camera sensors for a total $84^{\circ} \times 30^{\circ}$ FOV at an apogee of $7 \mathrm{R}_{\mathrm{E}}$. From inspection of Fig. 6, where the white circle represents the size of the Earth, a $10 \mathrm{R}_{\mathrm{E}}$ wide FOV, or $20^{\circ}$ × $20^{\circ}$ at $30 \mathrm{R}_{\mathrm{E}}$ geocentric distance, is

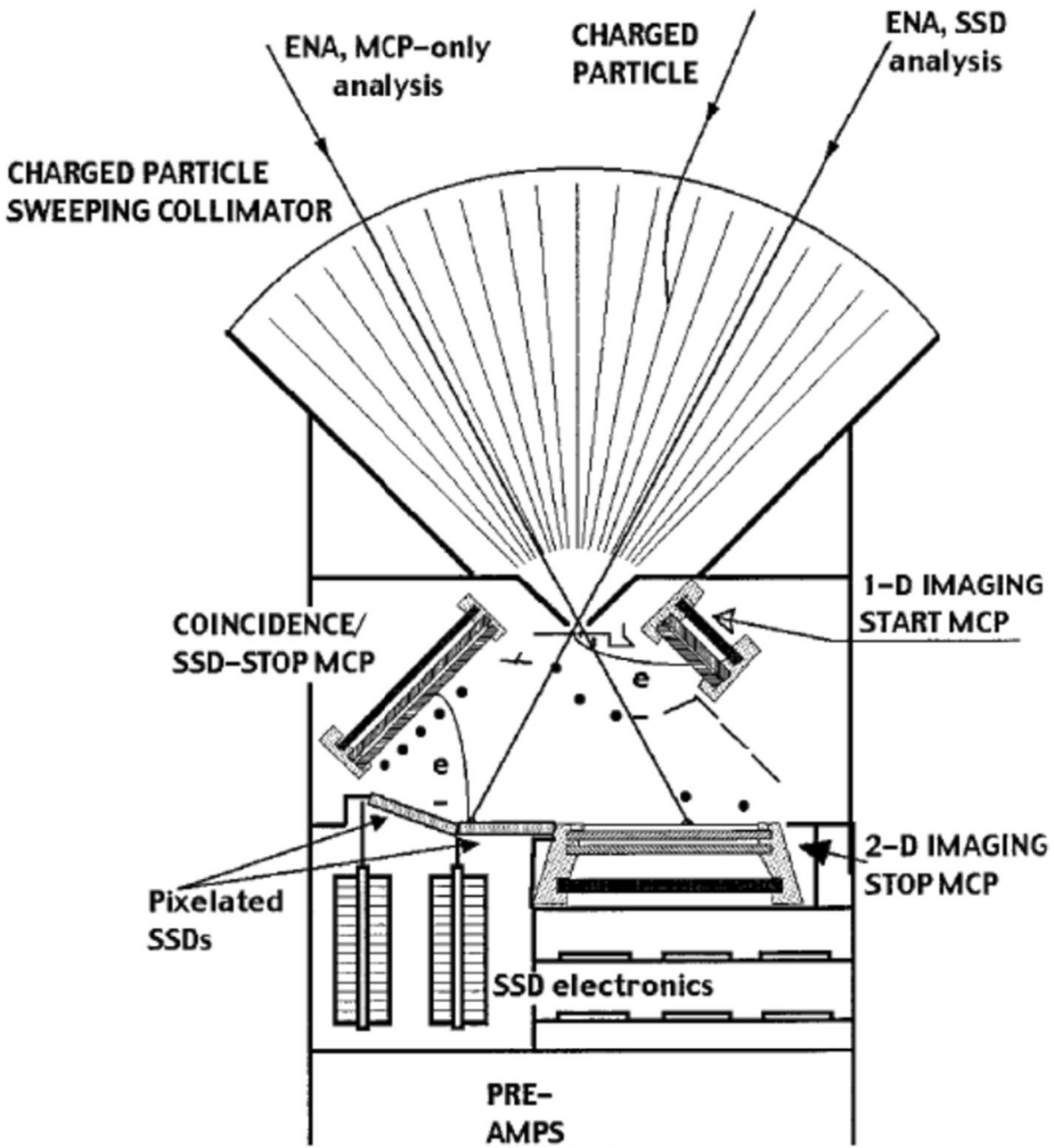

Fig. 11 IMAGE HENA instrument schematic (Mitchell et al. 2000) 
appropriate in order to provide, in a single exposure, a map of the entire plasmasphere from the outside, including plasmasphere plumes. A spatial resolution of $\sim 0.3 \mathrm{R}_{\mathrm{E}}$ and a 10 min cadence are necessary in order to satisfy science requirements.

Taking the design of IMAGE EUV (Sandel et al. 2000 - see Fig. 12) as an example, the entrance aperture of the imager incorporates an Aluminium filter that transmits the $\mathrm{He}^{+} 30.4 \mathrm{~nm}$ line, while excluding the bright geocoronal H Ly $\alpha$ line at $121.6 \mathrm{~nm}$. Light that goes through the filter reaches the mirror, which has a multilayer coating to provide good reflectivity at the target wavelength and low at $58.4 \mathrm{~nm}$ (this He I emission is expected to be weak in the plasmasphere, but can be quite bright in Earth's ionosphere). The mirror focuses the light on a sensor comprising bare microchannel plates (avoiding a photocathode that could increase the response to the contaminating $\mathrm{H} \mathrm{Ly} \alpha$ line) and a wedge and strip readout.

\subsubsection{In situ measurement package}

An in situ package, comprising a top-hat plasma analyser (e.g. measuring protons and $\alpha$-particles) and a magnetometer, completes the instrument suite, making the mission self-sufficient, i.e. avoiding the need to rely on other spacecraft to monitor solar wind/magnetosheath plasma conditions. Moreover, simultaneous in situ measurements in both hemispheres (and on dawn and dusk flanks) allow the study of magnetospheric asymmetries. If one of the satellites is upstream of the quasi-parallel bow shock and the other is upstream of the quasi-perpendicular bow shock, we can compare properties of the plasma, waves, and magnetic field parameters.

There is extensive heritage of in situ instrumentation on many previous and current space missions, e.g. Cluster and Cassini, THEMIS and MMS. The driver for the magnetometer is the determination of IMF discontinuities, and for the plasma analyser the detection of solar wind plasma $(0.5-4.0 \mathrm{keV})$ variations at cadences with sufficient energy resolution to construct moments. A broader energy range (e.g. 0.05-40 keV) would be required in order to characterise plasma in the magnetosheath, magnetotail,

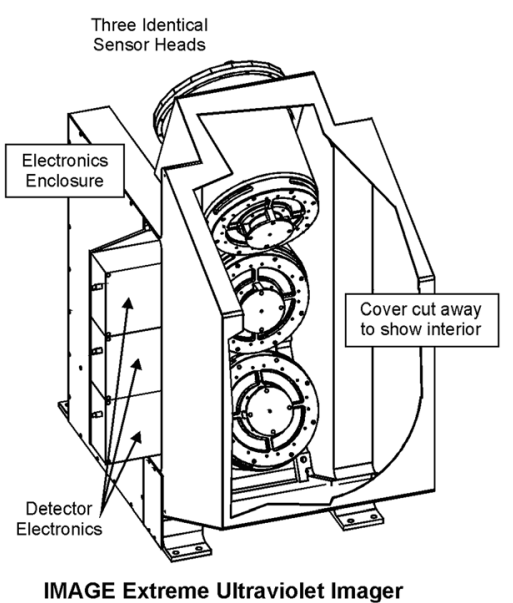

IMAGE Extreme Ultraviolet Imager

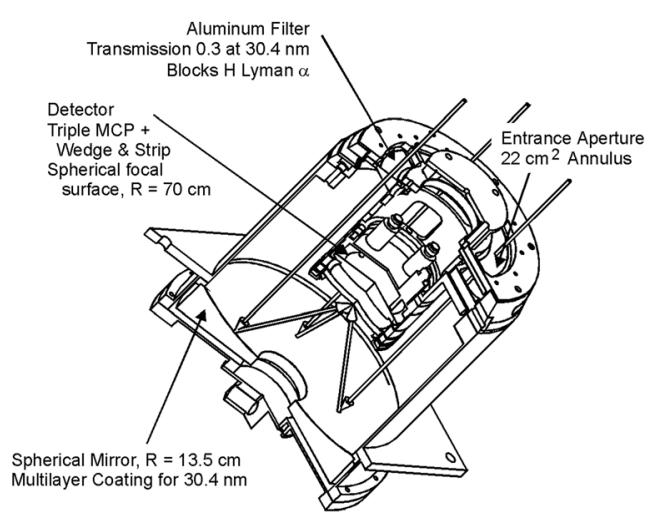

EUV Sensor Head (cutaway view)

Fig. 12 IMAGE EUV instrument. Left: Front view, with a portion of the main light baffle cut away to show the location of the sensor heads Right: Cutaway drawing of a sensor head (from Sandel et al. 2000) 
and the plasma sheet. Instrument requirements which follow are a resolution of $0.2 \mathrm{nT}$ at a cadence of $1.5 \mathrm{~s}$ for the fluxgate magnetometer and $\Delta \mathrm{E} / \mathrm{E}=0.2$ at sub-second cadence for the plasma analyser. These are easily satisfied by the type of magnetometers and plasma analysers flying on Cluster and Cassini, THEMIS and MMS, and Solar Orbiter.

\subsubsection{Additional observing facilities}

A strong link with ground-based measurement facilities, such as auroral imagers, magnetometer networks, and global networks of ionospheric radars, will be essential in order to e.g. closely examine beading features detected from space; determine the onset of substorms, which will be then followed in detail with the space instrumentation; make timing measurements between a solar wind discontinuity reaching the subsolar magnetopause and the ground responding; provide measurements of convection; and quantify reconnection rates. A space - ground facilities collaborative link of this kind has already been deployed and operated extensively in the context of the THEMIS mission, and is already planned for SMILE, so there are established precursors of the concepts described here.

Modelling support will also be required for the mission, in terms of both MHD and kinetic models. Again, this is an area under active development in preparation for the SMILE mission.

\section{Which technology challenges would enable addressing the science questions proposed?}

The proposed mission concept does not involve technological challenges in order to bear fruit: the instruments under consideration have already been flown or are at high TRL (Technology Readiness Level) in view of forthcoming launches. A single spacecraft version of the concept mission outlined above has already been studied in detail in preparation of previous mission proposals. The two spacecraft mission configuration is estimated to be within the envelope of an ESA M-class mission on the basis of resource studies carried out by the NASA GSFC members of this proposal team.

It must be stressed that even in the unfortunate case of one spacecraft failing, the multi-faceted nature of the science goals described will produce breakthroughs in our understanding of solar wind - magnetosphere coupling, and its impact on geospace and the human domain. Strong synergy will exist with other space missions (e.g. at L1 and L5) and ground measurements likely to take place in the medium term to 2050. Moreover, since the spacecraft will spend most of the time in the solar wind, while often visiting the magnetosheath, magnetotail, and the lobes, there will be the opportunity of carrying out a variety of other investigations of plasma conditions (e.g. turbulence, if in situ instrumentation with higher cadence could be part of the payload) making geospace an unparalleled plasma physics laboratory. Taking a larger scale holistic view, a mission such as the one envisaged here could represent the Earth-oriented component of an L-class mission incorporating elements linking solar, interplanetary, magnetospheric, and ionospheric exploration, a truly global observatory of the heliosphere, with science 
aims perfectly aligned with those of crucial and still underdeveloped space weather research.

\section{Conclusions}

In this paper we propose a tomographic imaging approach as the next step-up in the quest for a complete understanding of how the Sun gives rise to and controls the Earth's space plasma environment. In doing this we combine remote global imaging (soft Xray, FUV, EUV, ENA) and in situ probing of the solar wind and the magnetospheric plasmas. Our proposal uniquely links local space plasma with other planetary and astrophysical plasmas through the atomic physics process that occurs in all of them: charge exchange. In common with a number of scientists looking forward to the next decades of space research, we aim to achieve an understanding of the fundamental plasma processes taking place in the Universe, in our case by probing our local laboratory, geospace. Our aim is to establish the physical drivers of space weather affecting the Earth, to attempt to understand how we may be able to mitigate its effects and ultimately, forecast them.

Acknowledgements GBR and AAS acknowledge support by the UK Space Agency under grant ST/ T002964/1. YVB is supported by the STFC RAL Space In-house research grant. EAK is supported by the German Research Foundation (DFG) under number KR 4375/2 - 1 within SPP 'Dynamic Earth'. KML, KO and $\mathrm{N} \varnothing$ acknowledge financial support by the Research Council of Norway under the contract 223,252. SEM is supported by the Science and Technology Facilities Council (STFC), UK, grant no. ST/S000429/1.

Open Access This article is licensed under a Creative Commons Attribution 4.0 International License, which permits use, sharing, adaptation, distribution and reproduction in any medium or format, as long as you give appropriate credit to the original author(s) and the source, provide a link to the Creative Commons licence, and indicate if changes were made. The images or other third party material in this article are included in the article's Creative Commons licence, unless indicated otherwise in a credit line to the material. If material is not included in the article's Creative Commons licence and your intended use is not permitted by statutory regulation or exceeds the permitted use, you will need to obtain permission directly from the copyright holder. To view a copy of this licence, visit http://creativecommons.org/licenses/by/4.0/.

\section{References}

Akasofu, S.-I.: Auroral substorms: Search for processes causing the expansion phase in terms of the electric current approach. Space Sci. Rev. 212, 341-381 (2017)

Angelopoulos, V.: The THEMIS mission. Space Sci. Rev. 141, 5-34 (2008)

Angelopoulos, V., McFadden, J.P., Larson, D., Carlson, C.W., Mende, S.B., Frey, H., Phan, T., Sibeck, D.G., Glassmeier, K.-H., Auster, U., Donovan, E., Mann, I.R., Rae, I.J., Russell, C.T., Runov, A., Xhou, X., Kepko, L.: Tail reconnection triggering substorm onset. Science. 321, 931-935 (2008)

Benkevich, L., Lyatsky, W., Cogger, L.L.: Field-aligned currents between conjugate hemispheres. J. Geophys. Res. 105, 27727 (2000)

Borovsky, J.E., Denton, M.H.: Differences between CME-driven storms and CIR-driven storms. J. Geophys. Res. 111, A07S08 (2006)

Borovsky, J.E., Denton, M.H.: A statistical look at plasmaspheric drainage plumes. J. Geophys. Res. 113, A09221 (2008)

Boudouridis, A., Lyons, L.R., Zesta, E., Ruohoniemi, J.M.: Dayside reconnection enhancement resulting from a solar wind dynamic pressure increase. J. Geophys. Res. 112, A06201 (2007) 
Brambles, O.J., Lotko, W., Zhang, B., Wiltberger, M., Lyon, J., Strangeway, R. J.: Magnetosphere sawtooth oscillations induced by ionospheric outflow. Science, 332, 1183-1186 (2011)

Branduardi-Raymont, G., et al.: SMILE definition study report, European Space Agency, ESA/SCI, 1 (2018). https://doi.org/10.5270/esa.smile.definition_study_report-2018-12

Burch, J.L.: IMAGE mission overview. Space Sci. Rev. 91, 1-14 (2000)

Cargill, P.J., Lavraud, B., Owen, C.J., Grison, B., Dunlop, M.W., Cornilleau-Wehrlin, N., Escoubet, C.P., Paschmann, G., Phan, T.D., Rezeau, L., Bogdanova, Y., Nykyri, K.: Cluster at the magnetospheric cusps. Space Sci. Rev. 118, 321-366 (2005)

Carter, J.A., Milan, S.E., Fear, R.C., Walach, M.-T., Harrison, Z.A., Paxton, L.J., Hubert, B.: Transpolar arcs observed simultaneously in both hemispheres. J. Geophys. Res. 122, 6107-6120 (2017)

Carter, J.A., Milan, S.E., Fogg, A.R., Sangha, H., Lester, M., Paxton, L.J., Anderson, B.: The evolution of long duration cusp spot emission during lobe reconnection with respect to field-aligned currents. J. Geophys. Res. Space Physics 125, e2020JA027922 (2020)

Cassak, P.A., Shay, M.A.:: Scaling of asymmetric magnetic reconnection: General theory and collisional simulations. Phys. Plasmas. 14, 102114-102125 (2007)

Chisham, G., Lester, M., Milan, S.E., Freeman, M.P., Bristow, W.A., Grocott, A., McWilliams, K.A., Ruohoniemi, J.M., Yeoman, T.K., Dyson, P.L., Greenwald, R.A., Kikuchi, T., Pinnock, M., Rash, J.P.S., Sato, N., Sofko, G.J., Villain, J.-P., Walker, A.D.M.: A decade of the Super Dual Auroral Radar Network (SuperDARN): scientific achievements, new techniques and future directions. Surv. Geophys. 28, 33-109 (2007)

Cowley, S.W.H.: The causes of convection in the Earth's magnetosphere: A review of developments during the IMS. Rev. Geophys. Space Phys. 20, 531-565 (1982)

Daglis, I.A., Thorne, R.M., Baumjohann, W., Orsini, S.: The terrestrial ring current: origin, formation and decay. Rev. of Geophys. 37, 407-438 (1999)

DeJong, A.D., Ridley, A.J., Cai, X., Clouer, C.R.: A statistical study of BRIs (SMCs), isolated substorms, and individual sawtooth injections. J. Geophys. Res. 114, A08215 (2009)

Denton, M.H., Borovsky, J.E., Skoug, R.M., Thomsen, M.F., Lavraud, B., Henderson, M.G., McPherron, R.L., Zhang, J.C., Liemohn, M.W.: Geomagnetic storms driven by ICME- and CIR-dominated solar wind, J. Geophys. Res. 111, A07S07 (2006)

Dungey, J.W.: Interplanetary magnetic field and the auroral zones. Phys. Rev. Lett. 6, 47-48 (1961)

Eastwood, J.P., Hietala, H., Toth, G., Phan, T.D., Fujimoto, M.: What controls the structure and dynamics of Earth's magnetosphere? Space Sci. Rev. 188, 251-286 (2015)

Frey, H.U., Mende, S.B., Immel, T.J., Fuselier, S.A., Claflin, E.S., Gerard, J.C., Hubert, B.: Proton aurora in the cusp. J. Geophys. Res. 107, 1091-1107 (2002)

Goldstein, J., McComas, D.J.: Five years of stereo magnetospheric imaging by TWINS. Space Sci. Rev. 180, 39-70 (2013)

Gonzalez, W.D., Tsurutani, B.T., Gonzalez, A.L.C., Smith, E.J., Tang, F., Akasofu, S.-I.: Solar wind magnetosphere coupling during intense magnetic storms (1978-1979). J. Geophys. Res. 94, 88358851 (1989)

Gordeev, E., Sergeev, V., Honkonen, I., Kuznetsova, M., Rastätter, L., Palmroth, M., Janhunen, P., Tóth, G., Lyon, J., Wiltberger, M.: Assessing the performance of community-available global MHD models using key system parameters and empirical relationships. Space Weather (2015). https://doi.org/10.1002/ 2015SW001307

Hamilton, D.C., Gloeckler, G., Ipavich, F.M., Stüdemann, W., Wilken, B., Kremser, G.: Ring current development during the great geomagnetic storm of February 1986. J. Geophys. Res. 93, 14343-14355 (1988)

Herrera, D., Maget, V.F., Sicard-Piet, A.: Characterizing magnetopause shadowing effects in the outer electron radiation belt during geomagnetic storms. J. Geophys. Res. 121, 9517-9530 (2016)

Hsu, T.-S., McPherron, R.L.: Average characteristics of triggered and non-triggered substorms. J. Geophys. Res. 109, A07208 (2004)

Hubert, B., Gérard, J.-C., Milan, S.E., Cowley, S.W.H.: Magnetic reconnection during steady magnetospheric convection and other magnetospheric modes. Ann. Geophys. 35, 505-524 (2017)

Keller, K.A., Fok, M.-C., Narock, A., Hesse, M., Rastaetter, L., Kuznetsova, M.M., Gombosi, T.I., DeZeeuw, D.L.: Effect of multiple substorms on the buildup of the ring current. J. Geophys. Res. 110, A08202 (2005)

Laundal, K.M., Østgaard, N.: Asymmetric auroral intensities in the Earth's Northern and Southern hemispheres. Nature. 460, 491-493 (2009) 
Liemohn, M.W., Kozyra, J.U., Thomsen, M.F., Roeder, J.L., Lu, G., Borovsky, J.E., Cayto, T.E.: Dominant role of the asymmetric ring current in producing the stormtime Dst*. J. Geophys. Res. 106, 10883-10904 (2001)

Liemohn, M., Ganushkina, N.Y., De Zeeuw, D.L., Rastaetter, L., Kuznetsova, M., Welling, D.T., Toth, G., Ilie, R., Gombosi, T.I., van der Holst, B.: Real-time SWMF at CCMC: Assessing the Dst output from continuous operational simulations. Space Weather 16, 1583-1603 (2018)

Liou, K., Newell, P.T., Meng, C.-I., Wu, C.-C., Lepping, R.P.: Investigation of external triggering of substorms with Polar ultraviolet imager observations. J. Geophys. Res. 108, 1364-1377 (2003)

Liu, Y.D., Hu, H., Wang, R., Yang, Z., Zhu, B., Liu, Y.A., Luhmann, J.G., Richardson, J.D.: Plasma and magnetic field characteristics of solar coronal mass ejections in relation to geomagnetic storm intensity and variability. Astrophys. J. Lett. 809, L34-L39 (2015)

Luo, H., Kronberg, E.A., Nykyri, K., Trattner, K.J., Daly, P.W., Chen, G.X., Du, A.M., Ge, Y.S.: IMF dependence of energetic oxygen and hydrogen ion distributions in the near-Earth magnetosphere. J. Geophys. Res. 122, 5168-5180 (2017)

McComas, D.J., et al.: (40 co-authors): The Two Wide-angle Imaging Neutral-atom Spectrometers (TWINS) NASA Mission-of-Opportunity. Space Sci. Rev. 142, 157-231 (2009)

McFadden, J.P., Carlson, C.W., Larson, D., Bonnell, J., Mozer, F.S., Angelopoulos, V., Glassmeier, K.-H., Auster, U.: Structure of plasmaspheric plumes and their participation in magnetopause reconnection: First results from THEMIS. Geophys. Res. Lett. 35, L17S10 (2008)

McPherron, R.L.: Introduction to space physics. In: Kivelson, M.G., Russell, C.T. (eds.) Cambridge University Press, Cambridge (1995)

McPherron, R.L., Weygand, J.M., Hsu, T.-S.: Response of the Earth's magnetosphere to changes in the solar wind. J. Atmos. Solar Terr. Phys. 70, 303-315 (2008)

Mende, S.B., Heetderks, H., Frey, H.U., Stock, J.M., Lampton, M., Geller, S.P., Abiad, R., Siegmund, O.H.W., Habraken, S., Renotte, E., Jamar, C., Rochus, P., Gerard, J.-C., Sigler, R., Lauche, H.: Far ultraviolet imaging from the Image spacecraft. 3. Spectral imaging of Lyman- $\alpha$ and OI $135.6 \mathrm{~nm}$. Space Sci. Rev. 91, 287-318 (2000)

Milan, S.E.: Both solar wind-magnetosphere coupling and ring current intensity control of the size of the auroral oval. Geophys. Res. Lett. 36, L18101 (2009)

Milan, S.E., Lester, M., Cowley, S.W.H., Brittnacher, M.: Dayside convection and auroral morphology during an interval of northward interplanetary magnetic field. Ann. Geophys. 18, 436-444 (2000)

Milan, S.E., Hubert, B., Grocott, A.: Formation and motion of a transpolar arc in response to dayside and nightside reconnection. J. Geophys. Res. 110, A01212 (2005)

Mitchell, D.G., Jaskulek, S.E., Schlemm, C.E., Keath, E.P., Thomson, R.E., Tossman, B.E., Boldt, J.D., Hayes, J.R., Andrews, G.B., Paschalidis, N., Hamilton, D.C., Lundgren, R.A., Tums, E.O., Wilson, P.I.V., Voss, H.D., Prentice, D., Hsieh, K.C., Curtis, C.C., Powell, F.R.: High energy neutral atom (hena) imager for the IMAGE mission. Space Sci. Rev. 91, 67-112 (2000)

Mitchell, D.G., Hsieh, K.C., Curtis, C.C., Hamilton, D.C., Voss, H.D., Roelof, E.C., Brandt, P.C.: Imaging two geomagnetic storms in energetic neutral atoms. Geophys. Res. Lett. 28, 1151-1154 (2001)

Newell, P.T., Gjerloev, J.W.: Evaluation of SuperMAG auroral electrojet indices as indicators of substorms and auroral power. J. Geophys. Res. 116, A12211 (2011)

Østgaard, N., Mende, S.B., Frey, H.U., Frank, L.A., Sigwarth, J.B.: Observations of non-conjugate theta aurora. Geophys. Res. Lett. 30, 2125-2128 (2003)

Otto, A., Fairfield, D.H.: Kelvin-Helmholtz instability at the magnetotail boundary: MHD simulation and comparison with Geotail observation. J. Geophys. Res. 105, 21175-21190 (2000)

Plaschke, F., Hietala, H., Archer, M., Blanco-Cano, X., Kajdic, P., Karlsson, T., Lee, S.H., Omidi, N., Palmroth, M., Roytershteyn, V., Schmid, D., Sergeev, V., Sibeck, D.: Jets downstream of collisionless shocks. Space Sci. Rev. 214, 81-157 (2018)

Pulkkinen, T.I., Palmroth, M., Koskinen, H.E.J., Laitinen, T.V., Goodrich, C.C., Merkin, V.G., Lyon, J.G.: Magnetospheric modes and solar wind energy coupling efficiency. J. Geophys. Res. 115, A03207 (2010)

Reidy, J.A., Fear, R.C., Whiter, D.K., Lanchester, B., Kavanagh, A.J., Paxton, L.J., Zhang, Y., Lester, M.: Multi-instrument observation of simultaneous polar cap auroras on open and closed magnetic field lines. J. Geophys. Res. 122, 4367-4386 (2017)

Richmond, A.D., Roble, R.G.: Electrodynamic effects of thermospheric winds from the NCAR Thermospheric General Circulation Model. J. Geophys. Res. 92, 12365-12376 (1987)

Roelof, E.C., Brand, P.C., Mitchell, D.G.: Derivation of currents and diamagnetic effects from global plasma pressure distributions obtained by IMAGE/HENA. Adv. Space Res. 33, 747-751 (2004)

Runge, J., Balasis, G., Daglis, I.A., Papadimitriou, C., Donner, R.V.: Common solar wind drivers behind magnetic storm - magnetospheric substorm dependency. Nature 8, 16987-16996 (2018) 
Russell, C.T., Elphic, R.C.: Initial ISEE magnetometer results: Magnetopause observations. Space Sci. Rev. 22, 681-715 (1978)

Samsonov, A.A., Sibeck, D.G., Dmitrieva, N.P., Semenov, V.S.: What happens before a southward IMF turning reaches the magnetopause? Geophys. Res. Lett. (2017) https://doi.org/10.1002/2017GL075020

Sandel, B.R., Broadfoot, A., Curtis, C., King, R., Stone, T., Hill, R., Chen, J., Siegmund, O., Raffanti, R., Allred, D., Turley, R., Gallagher, D.: The extreme ultraviolet imager investigation for the IMAGE mission. Space Sci. Rev. 91, 197-242 (2000)

Sandel, B.R., Goldstein, J., Gallagher, D.L., Spasojevic, M.: Extreme ultraviolet imager observations of the structure and dynamics of the plasmasphere. Space Sci. Rev. 109, 25-46 (2003)

Sergeev, V.A., Pellinen, R.J., Pulkkinen, T.I.: Steady Magnetospheric convection: A review of recent results. Space Sci. Rev. 75, 551-604 (1996)

Shue, J.-H., Song, P., Russel, C.T., Steinber, J.T., Chao, J.K., Zastenker, G., Vaisberg, O.L., Kokubun, S., Singer, H.J., Detman, T.R., Kawano, H.: Magnetopause location under extreme solar wind conditions. J. Geophys. Res. 103, 17691-17700 (1998)

Shukhtina, M.A., Dmitrieva, N.P., Popova, N.G., Sergeev, V.A., Yahnin, A.G., Despirak, I.V.: Observational evidence of the loading-unloading substorm scheme. Geophys. Res. Lett. 32, L17107 (2005)

Sibeck, D.G., et al.: (34 co-authors): Imaging plasma density structures in the soft X-rays generated by solar wind charge exchange with neutrals. Space Sci. Rev. 214, 79-202 (2018)

Siscoe, G.L., Crooker, N.U., Erickson, G.M., Sonnerup, B.U.O., Maynard, N.C., Schoendorf, J.A., Siebert, K.D., Weimer, D.R., White, W.W., Wilson, G.R.: MHD properties of magnetosheath flow. Planet. Space Sci. 50, 461-471 (2002)

Sonnerup, B.U.O., Paschmann, G., Papamastorakis, I., Sckopke, N., Haerendel, G., Bame, S.J., Asbridge, J.R., Gosling, J.T., Russell, C.T.: Evidence for magnetic field reconnection at the Earth's magnetopause. J. Geophys. Res. 86, 10049 (1981)

Takahashi, S., Iyemori, T., Takeda, M.: A simulation of the storm-time ring current. Planet. Space Sci. 38, 1133-1141 (1990)

Taylor, M.G.G.T., et al.: (25 co-authors): The plasma sheet and boundary layers under northward IMF: A multi-point and multi-instrument perspective. Adv. Space Res. 41, 1619-1629 (2008)

Trattner, K.J., Mulcock, J.S., Petrinec, S.M., Fuselier, S.A.: Location of the reconnection line at the magnetopause during southward IMF conditions. Geophys. Res. Lett. 34, L03108 (2007)

Troshichev, O., Janzhura, A., Stauning, P.: Unified PCN and PCS indices: Method of calculation, physical sense, and dependence on the IMF azimuthal and northward components. J. Geophys. Res. 111, A05208 (2006)

Tsyganenko, N.A.: Data-based modelling of the Earth's dynamic magnetosphere: a review. Ann. Geophys. 31, 1745-1772 (2013)

Turner, D.L., Angelopoulos, V., Li, W., Hartinger, M.D., Usanova, M., Mann, I.R., Bortnik, J., Shprits, Y.: On the storm-time evolution of relativistic electron phase space density in Earth's outer radiation belt. J. Geophys. Res. 118, 2196-2212 (2013)

Walach, M.T., Milan, S.E., Murphy, K.R., Carter, J.A., Hubert, B.A., Grocott, A.: Comparative study of largescale auroral signatures of substorms, steady magnetospheric convection events, and saw-tooth events. J. Geophys. Res. 122, 6357-6373 (2017)

Wild, J.A., Woodfield, E.E., Morle, S.K.: On the triggering of auroral substorms by northward turnings of the interplanetary magnetic field. Ann. Geophys. 27, 3559-3570 (2009)

Publisher's Note Springer Nature remains neutral with regard to jurisdictional claims in published maps and institutional affiliations. 


\section{Affiliations}

G. Branduardi-Raymont ${ }^{1}$ - M. Berthomier ${ }^{2}$ - Y. V. Bogdanova ${ }^{3}$ - J. A. Carter ${ }^{4}$ M. Collier ${ }^{5}$ - A. Dimmock ${ }^{6}$ - M. Dunlop ${ }^{3,7} \cdot$ R. C. Fear ${ }^{8}$ - C. Forsyth ${ }^{1}$ - B. Hubert ${ }^{9}$. E. A. Kronberg ${ }^{10} \cdot$ K. M. Laundal ${ }^{11} \cdot$ M. Lester ${ }^{4}$. S. Milan ${ }^{4} \cdot$ K. Oksavik ${ }^{11}$. N. Østgaard ${ }^{11}$ - M. Palmroth ${ }^{12}$. F. Plaschke ${ }^{13}$. F. S. Porter ${ }^{5}$. I. J. Rae ${ }^{14}$. $^{\circ}$ A. Read $^{4}$ - A. A. Samsonov ${ }^{1}$ - S. Sembay ${ }^{4}$ - Y. Shprits ${ }^{15}$ - D. G. Sibeck ${ }^{5}$. B. Walsh ${ }^{16} \cdot$ M. Yamauchi ${ }^{17}$

\section{G. Branduardi-Raymont} g.branduardi-raymont@ucl.ac.uk

1 Mullard Space Science Laboratory, University College London, Holmbury St Mary, Dorking, Surrey RH5 6NT, UK

2 Laboratoire de Physique des Plasmas, Paris, France

3 Rutherford Appleton Laboratory, Didcot, UK

4 University of Leicester, Leicester, UK

5 NASA Goddard Space Flight Center, Greenbelt, MD, USA

6 Swedish Institute of Space Physics, Uppsala, Sweden

7 School of Space and Environment, Beihang University, Beijing, People's Republic of China

8 University of Southampton, Southampton, UK

9 University of Liege, Liege, Belgium

10 University of Munich, Munich, Germany

11 University of Bergen, Bergen, Norway

12 University of Helsinki, Helsinki, Finland

13 Space Research Institute, Austrian Academy of Sciences, Graz, Austria

14 Northumbria University, Newcastle upon Tyne, UK

15 German Research Centre for Geosciences, Potsdam, Germany

16 Boston University, Boston, MA, USA

17 Swedish Institute of Space Physics, Kiruna, Sweden 